LAWRENCE LIVERMORE NATIONAL LABORATORY

Neutron and Charged-Particle Induced Cross Sections for Radiochemistry in the Region of Bromine and Krypton

R. Hoffman, F. Dietrich, R. Bauer, K. Kelley, M. Mustafa

July 29, 2004 


\section{Disclaimer}

This document was prepared as an account of work sponsored by an agency of the United States Government. Neither the United States Government nor the University of California nor any of their employees, makes any warranty, express or implied, or assumes any legal liability or responsibility for the accuracy, completeness, or usefulness of any information, apparatus, product, or process disclosed, or represents that its use would not infringe privately owned rights. Reference herein to any specific commercial product, process, or service by trade name, trademark, manufacturer, or otherwise, does not necessarily constitute or imply its endorsement, recommendation, or favoring by the United States Government or the University of California. The views and opinions of authors expressed herein do not necessarily state or reflect those of the United States Government or the University of California, and shall not be used for advertising or product endorsement purposes.

\section{Auspices Statement}

This work was performed under the auspices of the U.S. Department of Energy by University of California, Lawrence Livermore National Laboratory under Contract W-7405-Eng-48. 
UCRL-TR-205563

\title{
Neutron and Charged-Particle Induced Cross Sections for Radiochemistry in the Region of Bromine and Krypton
}

\author{
R. D. Hoffman, F. S. Dietrich and R. Bauer \\ Nuclear Theory and Modeling Group \\ Physics and Advanced Technologies, N-Division \\ Lawrence Livermore National Laboratory \\ Livermore, CA 94550 \\ rdhoffman@llnl.gov \\ K. Kelley ${ }^{1}$ and M. Mustafa \\ Defense and Nuclear Technologies, AX-Division \\ Lawrence Livermore National Laboratory \\ Livermore, CA 94550
}

\begin{abstract}
We have developed a set of modeled nuclear reaction cross sections for use in radiochemical diagnostics. Systematics for the input parameters required by the Hauser-Feshbach statistical model were developed and used to calculate neutron and proton induced nuclear reaction cross sections in the mass region of bromine and krypton $(34 \leq \mathrm{Z} \leq 37,40 \leq \mathrm{N} \leq 47)$.
\end{abstract}

Subject headings: Nuclear cross sections, Radiochemistry, Nuclear Physics

\section{Introduction}

\subsection{Radiochemistry}

Various aspects of nuclear explosive device performance can be determined through the use of radiochemistry. During the UGT (Under Ground Test) Program, select naturally occurring elements were loaded into a device prior to a test and their activation products subsequentally retrieved for counting, typically with gamma-ray detectors. The products are measured as isotopic ratios (such as ${ }^{87} \mathrm{Y} /{ }^{88} \mathrm{Y}$ produced from a stable isotope of the naturally occurring element). From the measured activity and prior knowledge of the amount

\footnotetext{
${ }^{1}$ Department of Physics, University of California, Davis Davis, CA 95616
}

of loaded detector material, performance aspects could be inferred by comparing the measured isotope ratios with those calculated using neutron and charged-particle fluences from one of the design codes and group-averaged cross section sets that have been prepared for this purpose.

This paper details a collaborative effort between AX-Division and N-Division (PAT) to update and improve the existing charged particle cross section detector sets for the titanium (Ti), chromium $(\mathrm{Cr})$, bromine $(\mathrm{Br})$, iodine $(\mathrm{I})$, and europium $(\mathrm{Eu})$ mass regions. This paper will be devoted to the bromine-krypton detector set. We restrict our discussion to unclassified data related to the modeling effort. A separate classified document will discuss Stockpile Stewardship applications. 


\section{Contents}

1 Introduction 1

1.1 Radiochemistry . . . . . . . . . . . 1

1.2 Bromine and Krypton Detector Sets 4

1.2.1 Current Detector Sets . . . 4

1.2.2 The Need for New Detector Sets ........... 4

1.2.3 Proposed New BromineKrypton Detector Set . . . 5

2 Nuclear Reaction Theory 5

2.1 Reaction Mechanisms ...... 5

2.2 Hauser-Feshbach Statistical Model 5

2.3 Width Fluctuations . . . . . . . 6

2.4 Pre-Compound Processes . . . . 7

2.5 The STAPRE Hauser-Feshbach Reaction Code . . . . . . . . . . 7

3 Inputs Required for the HauserFeshbach Model 7

3.1 Nuclear Structure Data . . . . . 7

3.1.1 Nuclear Masses and $J^{\pi}$ Assignments . . . . . . . 7

3.1.2 Nuclear Level Schemes . . . 7

3.2 Transmission Coefficients . . . . 7

3.2.1 Transmission Coefficients for Particles . . . . . . . . 7

3.2.2 The Optical Potential of Koning and Delaroche . . . 8

3.2.3 Evaluation of the Optical Potential . . . . . . . 8

3.2.4 Transmission Coefficients for Photons . . . . . . 8

3.3 Nuclear Level Densities . . . . . . 9

3.3.1 Level Density Models . . . 9

3.3.2 Level Densities Above the Neutron Binding Energy . . 10

The Spin Cutoff Parameter . . . . 10

Pairing Energies . . . . . . . . . 10

The Level Density Parameter . . . 10

Shell Corrections . . . . . . . . . 10

3.3.3 Systematic Behavior of Fermi Gas Level Density Parameters 11
3.3.4 Level Densities Below the Neutron Binding Energy . .

Behavior of the Spin Cutoff Parameter Below $E_{x} \ldots \ldots \ldots$

4 Calculated Cross Sections $\quad 13$

4.1 Comparison to Measured Cross Sections ............ 13

4.2 Sensitivity Studies . . . . . . . . . 14

4.2.1 Sensitivity to the Pre-Equilibrium Cross Section . . . . . . 15

4.2.2 Sensitivity to the choice of Level Density Prescription . 15

4.2.3 Sensitivity to the Normalization of the $\gamma$-ray Transmission Coefficient ..... 16

4.2.4 Sensitivity to the Width Fluctuation Correction . . . 16

4.3 Production and Destruction Cross Sections ........... 17

4.3.1 Results for Remaining Reactions in the Detector Set 17

$\begin{array}{lll}5 & \text { Conclusions } & 18\end{array}$

6 Acknowledgments 18

A Basic Nuclear Structure Data 20

A.1 New Se-Br-Kr Detector Set . . . 20

A.2 Binding and Separation Energies . 22

A.3 Q-values for Select Reactions ... 24

A.4 Adopted Level Schemes . . . . . 25

A.5 Nuclear Level Density Parameters 35

A.6 Modeled Cross Sections vs. Experiment ........... 37

A.7 Modeled Cross Sections: Production and Destruction Channels . . 42 


\section{List of Figures}

1 Total neutron and proton Cross Sections vs Koning-Delaroche . . .

2 Systematics for average total swave radiation width. . . . . . .

$3 \quad \chi^{2}$ linear fit to experimentally determined shell corrections, used to systematically determine unknown shell corrections. . . . . . . . . .

4 Sample of a typical fit to low lying spectroscopic levels . . . . . . . .

5 Calculated vs. Experimental Cross Sections: ${ }^{79} \mathrm{Br} . \quad \ldots \ldots \ldots$. . . .

6 Sensitivity to Pre-Equilibrium Matrix Element. . . . . . . . . . . . .

7 Sensitivity to the Level Density Prescription. . . . . . . . .

8 Sensitivity to the s-wave Average Photon Width. . . . . . . . . .

9 Sensitivity to the Width Fluctuation Correction. . . . . . . . . .

10 Calculated Cross Sections directly affecting production and destruction of ${ }^{79} \mathrm{Kr} . \ldots . . . . . .$.

11 Adopted level schemes for $\mathrm{Br}$ and Kr nuclei, $\mathrm{N}=40$. . . . . . . .

12 Adopted level schemes for $\mathrm{Br}$ and Kr nuclei, $\mathrm{N}=41 \ldots \ldots$. . . . .

13 Adopted level schemes for $\mathrm{Br}$ and $\mathrm{Kr}$ nuclei, $\mathrm{N}=42 \ldots \ldots$. . . .

14 Adopted level schemes for $\mathrm{Br}$ and Kr nuclei, $\mathrm{N}=43 \ldots \ldots$. . . . .

15 Adopted level schemes for $\mathrm{Br}$ and Kr nuclei, $\mathrm{N}=44$. . . . . . . .

16 Adopted level schemes for $\mathrm{Br}$ and Kr nuclei, $\mathrm{N}=45 \quad \ldots \ldots \ldots$

17 Adopted level schemes for $\mathrm{Br}$ and $\mathrm{Kr}$ nuclei, $\mathrm{N}=46 \quad \ldots \ldots \ldots . . \quad 32$

18 Adopted level schemes for $\mathrm{Br}$ and Kr nuclei, $\mathrm{N}=47 \ldots \ldots . . . \quad \ldots 33$

19 Adopted level schemes for $\mathrm{Br}$ and Kr nuclei, $\mathrm{N}=48 \ldots \ldots . . . \quad \ldots \quad 34$

20 Measured vs. calculated $(\mathrm{n}, \gamma)$ cross sections. . . . . . . . . . . .

21 Measured vs. calculated Maxwellian averaged $(\mathrm{n}, \gamma)$ cross sections. . . .

22 Measured vs. calculated $(\mathrm{n}, 2 \mathrm{n})$ cross sections. . . . . . . . . .

23 Measured vs. calculated $(\mathrm{n}, \mathrm{x})$ and $(\mathrm{p}, \mathrm{x})$ cross sections. . . . . . . . .

24 Modeled cross sections for production and destruction channels: $\mathrm{N}=41$ nuclei . . . . . . . . . . . . .

25 Modeled cross sections for production and destruction channels: $\mathrm{N}=42$ nuclei . . . . . . . . . . . .

26 Modeled cross sections for production and destruction channels: $\mathrm{N}=43$ nuclei . . . . . . . . . . .

27 Modeled cross sections for production and destruction channels: $\mathrm{N}=44$ nuclei . . . . . . . . . . .

28 Modeled cross sections for production and destruction channels: $\mathrm{N}=45$ nuclei . . . . . . . . . . .

29 Modeled cross sections for production and destruction channels: $\mathrm{N}=46$ nuclei . . . . . . . . . . .

30 Modeled cross sections for production and destruction channels: $\mathrm{N}=47$ nuclei . . . . . . . . . .

\section{List of Tables}

1 Cross sections available in previous detector sets Br0391 and Kr0991.

2 Reactions calculated for new $\mathrm{Br}-\mathrm{Kr}$ set. . . . . . . . . . . . 21

$3 J^{\pi}$, Binding, and Separation Energies for new Br-Kr set. . . . . . . 23

4 Q-values $(\mathrm{MeV})$ for select reactions in the new Br-Kr set. . . . . . . . . 24

5 Level density parameters calculated for new Br-Kr set. . . . . . . . . . 


\subsection{Bromine and Krypton Detector Sets}

\subsubsection{Current Detector Sets}

Over the last 40 years a number of detector sets have been developed at LLNL and LANL. Twenty-three neutron threshold detector sets and five charged particle sets are currently available. In this paper our interest centers on the production of the noble gas ${ }^{79} \mathrm{Kr}\left(\tau_{1 / 2}=1.455 \mathrm{~d}\right)$. Two sets, both developed in 1991, are available.

The bromine set (Br0391) is a charged particle set used to calculate activation of the above mentioned $\mathrm{Kr}$ isotope from stable ${ }^{79} \mathrm{Br}$ and ${ }^{81} \mathrm{Br}$. The bromine set consists of 17 reactions, four charged particle reactions based on experiment (West et al. 1993) and 13 neutron induced reactions based on calculations and estimates.

The krypton set (Kr0991) is a neutron threshold set consisting of seven reactions, and is used to calculate the production of ${ }^{79} \mathrm{Kr}$ by neutron induced reactions on stable ${ }^{78} \mathrm{Kr}$ and ${ }^{80} \mathrm{Kr}$. Four cross sections are based on experimental data. For both sets, the available reactions are summarized in Table 1. These sets can be accessed on the world wide web, see http://nuclear.llnl.gov/CNP/nads/main.html.

\begin{tabular}{|c|c|c|}
\hline Set $\rightarrow$ & Br0391 & Kr0991 \\
\hline Experimental & $\begin{array}{c}{ }^{79} \mathrm{Br}(\mathrm{p}, \mathrm{n}){ }^{79} \mathrm{Kr} \\
{ }^{81} \mathrm{Br}(\mathrm{p}, \mathrm{n}){ }^{81} \mathrm{Kr} \\
{ }^{79} \mathrm{Br}(\mathrm{d}, 2 \mathrm{n}){ }^{79} \mathrm{Kr} \\
{ }^{81} \mathrm{Br}(\mathrm{d}, 2 \mathrm{n}){ }^{81} \mathrm{Kr}\end{array}$ & $\begin{array}{c}{ }^{80} \mathrm{Kr}(\mathrm{n}, 2 \mathrm{n}){ }^{79} \mathrm{Kr} \\
{ }^{78} \mathrm{Kr}(\mathrm{n}, 2 \mathrm{n}){ }^{77} \mathrm{Kr} \\
{ }^{78} \mathrm{Kr}(\mathrm{n}, \gamma){ }^{79} \mathrm{Kr} \\
{ }^{80} \mathrm{Kr}(\mathrm{n}, \gamma){ }^{81} \mathrm{Kr}\end{array}$ \\
\hline Calculated & $\begin{array}{c}{ }^{79} \mathrm{Br}(\mathrm{t}, \mathrm{n}){ }^{81} \mathrm{Kr} \\
{ }^{80} \mathrm{Kr}(\mathrm{n}, 2 \mathrm{n}){ }^{79} \mathrm{Kr} \\
{ }^{79} \mathrm{Kr}(\mathrm{n}, \gamma){ }^{80} \mathrm{Kr} \\
{ }^{80} \mathrm{Kr}(\mathrm{n}, \gamma){ }^{81} \mathrm{Kr} \\
{ }^{81} \mathrm{Kr}(\mathrm{n}, \gamma){ }^{82} \mathrm{Kr}\end{array}$ & $\begin{array}{c}{ }^{79} \mathrm{Kr}(\mathrm{n}, 2 \mathrm{n}){ }^{78} \mathrm{Kr} \\
\left.{ }^{79} \mathrm{Kr} \text { (n,other }\right)\end{array}$ \\
\hline Estimated & $\begin{array}{c}{ }^{79} \mathrm{Br}(\mathrm{n}, 2 \mathrm{n}){ }^{78} \mathrm{Br} \\
{ }^{80} \mathrm{Br}(\mathrm{n}, 2 \mathrm{n}){ }^{79} \mathrm{Br} \\
{ }^{81} \mathrm{Br}(\mathrm{n}, 2 \mathrm{n}){ }^{80} \mathrm{Br} \\
{ }^{79} \mathrm{Kr}(\mathrm{n}, 2 \mathrm{n}){ }^{78} \mathrm{Kr} \\
{ }^{81} \mathrm{Kr}(\mathrm{n}, 2 \mathrm{n}){ }^{80} \mathrm{Kr} \\
{ }^{79} \mathrm{Br}(\mathrm{n}, \gamma){ }^{80} \mathrm{Br} \\
{ }^{80} \mathrm{Br}(\mathrm{n}, \gamma){ }^{81} \mathrm{Br} \\
{ }^{81} \mathrm{Br}(\mathrm{n}, \gamma){ }^{82} \mathrm{Br}\end{array}$ & ${ }^{79} \mathrm{Kr}(\mathrm{n}, \gamma){ }^{80} \mathrm{Kr}$ \\
\hline
\end{tabular}

Table 1: Cross sections available in previous detector sets Br0391 and Kr0991.

\subsubsection{The Need for New Detector Sets}

The motivations for revisiting the detector sets for radiochemistry are many. The current sets were often developed based on "best guess" and "experience" from a limited amount of experimental data over a 40 year period. Often a single measurement at $14 \mathrm{MeV}$ guided the evaluation of a critical $(n, 2 n)$ cross section, with an assumed shape that would rise from a calculated threshold, and then be adjusted to match the experimental point at $14 \mathrm{MeV}$. This was often applied to other targets to make up a set that covered a range of neutron numbers.

For the two sets of interest here (Br0391 and Kr0991), a total of nine reaction cross sections were estimated and/or extrapolated from data spanning energies up to $1 \mathrm{MeV}$. Many are still based on preliminary data. Additionally, in 1991, only eight cross sections could be compared to experimental data. For details, see (Nethaway 1998).

Of the remaining reactions, many were modeled or estimated, but often only over a limited energy range. Beyond this range they were extrapolated. For example, the $(\mathrm{n}, 2 \mathrm{n})$ reactions on ${ }^{79,80,81} \mathrm{Br}$ and ${ }^{79,81} \mathrm{Kr}$ were all estimated from assumed maximum cross sections and a standard shape rising from threshold. The (n, $\gamma)$ reactions on ${ }^{79,80,81} \mathrm{Br}$ were all estimated based on data in the BNL cross section compilation (Mclane Dunford \& Rose 1988) for ${ }^{79,81} \mathrm{Br}$ and elemental bromine, with educated guesses for extrapolations to both lower and higher energies. The $(\mathrm{n}, \gamma)$ cross sections for ${ }^{79,80,81} \mathrm{Kr}$ were all based on calculations (Walter 1986) over the energy range $1 \mathrm{keV}$ to $1 \mathrm{MeV}$. Above this range all the cross sections were extrapolated by assuming a value of $1 \mathrm{mb}$ at $20 \mathrm{MeV}$.

In the decade since these cross section sets were last evaluated many new cross section measurements have been performed, and several efforts have been made to develop consistent approaches to modeling nuclear reaction cross sections (RIPL 1998). The basic nuclear structure data has been greatly improved. Finally, there are more accurate methods of calculating and estimating cross sections for which we have no data. 


\subsubsection{Proposed New Bromine-Krypton Detector Set}

We consider as targets all isotopes of the elements $\mathrm{Se}, \mathrm{Br}, \mathrm{Kr}$, and $\mathrm{Rb}(34 \leq \mathrm{Z} \leq 37)$ with neutron numbers $40 \leq \mathrm{N} \leq 47$ (including any long-lived isomers with half-lives greater than 1 $\mu \mathrm{s})$, and have calculated nuclear reaction cross sections for incident neutrons and protons on these targets with laboratory incident particle energies ranging from $0.01 \mathrm{keV}$ to $20 \mathrm{MeV}$. These compound systems are then allowed to decay through the reaction channels shown in Table 2 (see the Appendix).

The reason for including many more isotopes than were included in the original sets is to account for the various possible destruction reactions that are significant in this mass range. In general, the current RADCHEM detector sets, and especially the charged-particle sets, were developed with special attention paid to the production reactions. We have also included the isomers as targets, which were not included in the original sets, in order to gauge the sensitivity of the set to their inclusion. The activation products ${ }^{79,81} \mathrm{Kr}$, as measured in the UGT Program, only consider decay to the ground states of these species. We actually delivered to A-Program two detector sets, the Activation set, which includes only reactions that couple the ground states of all the isotopes considered, and the Full set, which included the isomers as well.

Another important reason for considering a larger range of nuclei is to compare our calculated cross sections to the many measured cross sections available for the stable isotopes of selenium. Our goal is to develop a consistent set that reproduces, as closely as possible, measured cross sections on targets in the local region of interest. To do this we develop local systematics for the many input quantities that are included in the theoretical reaction modeling calculations. These systematics are based on experimental data that are often only available for compound nuclear systems formed from a stable target plus a neutron. Of course, we use experimental data whenever it is available, but reactions proceeding through unstable systems are unavoidable in radiochemistry. Short of developing new experimental techniques to measure cross sections on unstable targets, our only hope of re- producing measured activity from UGT shots, and addressing the uncertainty associated with the nuclear cross sections, is to develop cross section sets that reproduce well the measured cross sections in the region of interest.

In $\S 2$ we will describe the theoretical techniques used in the modeling effort. $\S 3$ describes the input parameters. $\S 4$ gives results. We conclude with $\S$ 5 .

\section{Nuclear Reaction Theory}

\subsection{Reaction Mechanisms}

Conceptually, we consider nuclear reaction mechanisms to be of two general types, direct processes and compound processes. Direct processes can be pictured as simple interactions of the incident particle with the nuclear potential of the target nucleus. They proceed on a rapid time scale (of order $\sim 10^{-22} \mathrm{~s}$ ), and the reaction products are often highly peaked in the incident particle direction. Compound processes are pictured as complicated interactions proceeding over a much longer timescale $\left(10^{-15}-10^{-18} \mathrm{~s}\right)$ in which the reaction is mediated by the formation of a "compound nucleus", with the excitation energy of the incident particle being statistically "shared" with the ensemble of nucleons in the target over all energetically allowed degrees of freedom. The reaction products are largely isotropic. Compound nuclear reactions proceed through resonances, which correspond to nuclear states above the bound region, while direct reactions proceed through smooth potential terms. Other intermediate reaction mechanisms may exist between these two extremes. We refer to these as "precompound" nuclear processes. Over the energy range of interest to this project, a few $\mathrm{keV}$ to 20 $\mathrm{MeV}$, we will consider pre-compound and compound nuclear processes, with the pre-compound reactions operating principally above $10 \mathrm{MeV}$ of incident excitation energy.

\subsection{Hauser-Feshbach Statistical Model}

A traditional theoretical approach to compound nuclear reactions is the statistical or HauserFeshbach model. This model is valid only for high level densities in the compound nucleus, allowing one to use energy averaged transmission coefficients $T$, which describe absorption via an imagi- 
nary part in the (optical) nucleon-nucleus potential (for details see Mahaux and Weidenmüller 1979). For the reaction I (in state $\mu$ ) $+j \rightarrow k+\mathrm{L}$ (in state $\nu$ ), with $\mathrm{I}^{\nu}+j$ interacting with centerof-mass energy $\mathrm{E}_{j}^{\mu}$ (in $\mathrm{MeV}$ ), the average cross section is given by

$$
\sigma_{j k}^{\mu \nu}\left(E_{j}^{\mu}\right)=\frac{\pi \lambda_{j}^{2}}{g_{I}^{\mu} g_{j}} \sum_{J, \pi} g_{J} \frac{T_{j}^{\mu}\left(J^{\pi}\right) T_{k}^{\nu}\left(J^{\pi}\right)}{T_{t o t}\left(J^{\pi}\right)} W\left(J^{\pi}\right)
$$

where the summation extends over all compound nuclear spins and parities $J^{\pi}, \mu$ and $\nu$ are states in the target and product $(=0$ for the ground state, 1 for the $1^{\text {st }}$ excited state, etc.). The cross section has units of area, described by $\pi \lambda_{j}^{2}=$ $0.6566\left(\hat{A}_{j} E_{j}^{\mu}\right)^{-1}$ barns, with $\hat{A}_{j}=\left(A_{I} A_{j}\right) /\left(A_{I}+\right.$ $A_{j}$ ) being the reduced mass in atomic mass units and $E_{j}^{\mu}$ is the center of mass energy in units of $\mathrm{MeV} . \lambda_{j}$ is the wavelength related to the wave number $k_{j}$ in the target plus incident particle channel by $\lambda_{j}=1 / k_{j}$ The statistical weights are given by $g_{y}^{x}=\left(2 J_{y}^{x}+1\right)$. Items without superscripts refer to the compound nucleus.

The transmission coefficients in the numerator are given by $T_{j}^{\mu}\left(J^{\pi}\right)=$ the total transmission function for forming the state $\mathrm{J}^{\pi}$ in the compound nucleus $I^{\mu}+j$ at energy $E_{j}^{\mu}$. Likewise, $T_{k}^{\nu}\left(J^{\pi}\right)$ is the same as $T_{j}^{\mu}\left(J^{\pi}\right)$ but for the pair $L^{\nu}+k$ at energy $E_{k}^{\nu}$. Implicit in these definitions is a sum over all possible $l$-waves and channel spins, i.e.

$$
T_{j}^{\mu}\left(J^{\pi}\right)=\sum_{l, s} T_{j}^{\mu}\left(J^{\pi}, l, s\right)
$$

where $l$ is any partial wave number (orbital angular momentum) that can couple the state $\mu$ to the compound nuclear state having spin and parity $J^{\pi}$ subject to quantum mechanical selection rules and $s$ is the vector sum of the spins $J_{I}^{\mu}$ and $J_{j}$. Hence $s$ takes on all integer (or half-integer) numbers from $\left|J_{I}^{\mu}-J_{j}\right|$ to $J_{I}^{\mu}+J_{j}$.

$T_{\text {tot }}$ represents the the sum of transmission coefficients over all possible decay channels (i.e. for all particles and photons). The cross section for the formation of species L, regardless of its state $\nu$, is obtained by summing Eq. [1] over all bound states $\nu$ of $\mathrm{L}$ for which the reaction is energetically allowed.

When evaluating these sums, if energies become of interest which exceed the highest discrete excited state for which energy, spin, and parity are explicitly known, a nuclear level density formula must be employed. Specifically, the definitions for $T_{j}\left(J^{\pi}\right), T_{k}\left(J^{\pi}\right)$, and $T_{t o t}\left(J^{\pi}\right)$ must be modified, for example:

$$
\begin{aligned}
& T_{k}\left(J^{\pi}\right)=\sum_{\nu=0}^{\omega} T_{k}^{\nu}\left(J^{\pi}\right)+ \\
& \int_{\xi_{L}^{\omega}}^{\xi_{L}^{\max }} \int_{J^{\nu} \pi^{\nu}} T_{k}^{\nu}\left(\xi_{L}^{\nu}, J^{\pi}\right) \rho\left(\xi_{L}^{\nu}, J^{\nu}, \pi^{\nu}\right) d \xi_{L}^{\nu} d \pi^{\nu} d J^{\nu}
\end{aligned}
$$

where for the nucleus $\mathrm{L}, \xi_{L}^{\omega}$ is the energy of the highest excited state, $\omega$, of known energy, spin, and parity; $\xi_{L}^{\max }=E_{k}^{0}=E_{j}^{0}+Q_{j k}$ is the maximum excitation energy available, and $\rho\left(\xi_{L}^{\nu}, J^{\nu}, \pi^{\nu}\right)$ is the density of states per unit energy of spin and parity $J^{\nu}$ and $\pi^{\nu}$ at the excitation energy $\xi_{L}^{\nu}$. The above integral approximates a summation and is subject to the same quantum mechanical restrictions implied in the definition of the transmission function.

\subsection{Width Fluctuations}

In addition to the ingredients required for Eq. [1], like the transmission coefficients for particles and photons or the level densities, width fluctuation corrections (WFC) have to be employed as well. They define the correlation factors with which all partial channels of incoming particle $j$ and outgoing particle $k$, passing through excited state $(E, J, \pi)$, have to be multiplied. The major effect is to enhance the elastic channel and accordingly decrease the other open channels. They are most often observed at or near channel opening energies when i.e. a $(\mathrm{p}, \gamma)$ and a $(\mathrm{p}, \mathrm{n})$ channel compete and the weaker $(\mathrm{p}, \gamma)$ channel is enhanced. Above a few $\mathrm{MeV}$ of excitation energy, when many competing channels are open, WFC's can be neglected.

The exact expression for the WFC, obtained with the Gaussian orthoganal ensemble (GOE) approach, requires the evaluation of a triple integral and to date has been considered much to costly to apply in nuclear cross section calculations. Several approximations have been developed, the most popular ones are the Moldauer model (Moldauer 1976), and the HRTW model (Hofmann et al. 1975). We use the Moldauer 
model approximation in this study. For a detailed description of the full (GOE) treatment and a comparison with the Moldauer and HRTW approximation models mantioned above, see (Hilaire Lagrange \& Koning 2003).

\subsection{Pre-Compound Processes}

For excitation energies starting around $10 \mathrm{MeV}$, pre-compound processes become important. The pre-compound cross section is subtracted from the total cross section of the first compound nucleus, and is usually unimportant for subsequent compound nuclei. Here we describe equilibration of the compound nuclear system in terms of a simple exciton model. In the pre-equilibrium stage of the reaction particle emission is assumed to be the only decay mode. For the equilibration portion of the first chance particle or photon emission as well as for first chance fission, the width fluctuated Hauser Feshbach formula (Eq. [1]) is applied. All subsequent (higher chance) processes are treated as sequential evaporation steps.

\subsection{The STAPRE Hauser-Feshbach Reac- tion Code}

We adopt the statistical model code STAPRE (STAatistical-PREequilibrium) to model our cross sections. (Uhl \& Strohmaier 1976). It embodies all of the physical models discussed above. The version of the code we use is STAPRE-H95 (Avrigeanu \& Avrigeanu 1976), available from the NEA web site. We have made several modifications, primarily to the level density routines. Prior versions of the code were used to develop parts of the existing RADCHEM data sets (Vonach 1982).

In the following we discuss the important ingredients of statistical model calculations, and the methods utilized to estimate them. These are the requisite nuclear structure data (energies, spins, and parities of the ground states and all known excited states, as well as detailed branching ratios for gamma-cascade from excited to lowlying states), the width fluctuation corrections, the pre-compound cross section, the particle and $\gamma$-transmission coefficients, and the nuclear level densities of all nuclei involved in the reaction. The reliability of such calculations is chiefly determined by the accuracy with which these components can be evaluated.

\section{Inputs Required for the Hauser-Feshbach Model}

\subsection{Nuclear Structure Data}

\subsubsection{Nuclear Masses and $J^{\pi}$ Assignments}

We adopt for nuclear masses the experimental mass excess values of (Möller et al. 1995). Spin and parity assignments are from the Evaluated Nuclear Structure Data File (ENSDF 2003). We present in Table 3 (see the Appendix) the binding energies (in $\mathrm{MeV}$ ) calculated from the adopted masses, the ground state spins and parities, and the separation energies for neutrons, protons, alpha-particles, and deuterons for the nuclei included in this study. In Table 4 (See the Appendix), we provide Q-values for various cross sections that were calculated in this study.

\subsubsection{Nuclear Level Schemes}

The nuclear structure data needed to model the gamma-ray cascade in this study was adopted from the file BUDAPEST.DAT (RIPL 1998). For the bromine and krypton isotopes, additional evaluation was performed by R. Bauer (Bauer 2002). Shown in Appendix A.4 are the modified adopted nuclear levels, including level energy, spin and parity assignments, and their gamma-cascade branching ratios. The number of levels adopted for each nucleus is given as the quantity "N" in Table 5 . For the $\mathrm{Se}$ and $\mathrm{Rb}$ isotopes, this was the number for which energy spin and parity were unambiguously assigned in the BUDAPEST file.

\subsection{Transmission Coefficients}

\subsubsection{Transmission Coefficients for Particles}

We restrict our attention in this modeling effort to reactions with incident neutrons and protons. For the charged particle cross sections to be included in the new Br-Kr set, we adopt the experimental results of West (West et al. 1993) for the reactions ${ }^{79} \mathrm{Br}(\mathrm{p}, \mathrm{n}){ }^{79} \mathrm{Kr},{ }^{81} \mathrm{Br}(\mathrm{p}, \mathrm{n}){ }^{81} \mathrm{Kr}$, ${ }^{79} \mathrm{Br}(\mathrm{d}, 2 \mathrm{n}){ }^{79} \mathrm{Kr}$, and ${ }^{81} \mathrm{Br}(\mathrm{d}, 2 \mathrm{n}){ }^{81} \mathrm{Kr}$. We do present modeled results for $(\mathrm{p}, \mathrm{n})$ and $(\mathrm{p}, 2 \mathrm{n})$ reactions on all included isotopes for elements $\mathrm{Se}, \mathrm{Br}$, and $\mathrm{Kr}$. Ideally, one would prefer $(\mathrm{d}, \mathrm{n})$ and $(\mathrm{d}, 2 \mathrm{n})$ cross sections on all targets in the set. However, for use as a radiochemical detector set, the omission is not important, as only ${ }^{79,81} \mathrm{Br}$ are loaded, 
and any abundance produced during activation will be so small that the impact on the deuterium abundance (compared to fusion reactions) will be negligible.

\subsubsection{The Optical Potential of Koning and De- laroche}

For the calculation of the particle transmission coefficients, we used the optical model developed by (Koning \& Delaroche 2001). Although they have tuned their parameters to fit data for many different species (see their Tables 6 and 7), we decided to use the Global nucleon-nucleon Optical Model Potential, as it gives a very satisfactory fit to measured total cross section data for neutrons and protons in the range of interest to us (e.g. As-Kr). In specific, we adopted the potential depth parameters and fermi energies for the neutron and proton global OMP defined in their Section 5.2, tables 14 and 15 . The particle transmission coefficients were generated by the optical model code ECIS-95 (Raynal 1996). Although designed for coupled channel calculations, we used the code in a spherical optical model mode.

\subsubsection{Evaluation of the Optical Potential}

We present in Figure (1) results of the Koning \& Delarouche optical model compared to measured total cross sections. The first two comparisons are for total neutron cross sections for ${ }^{75} \mathrm{As}$ and ${ }^{89} \mathrm{Y}$, the last one is a comparison to the ${ }^{75} \mathrm{As}(\mathrm{p}, \mathrm{n}){ }^{75} \mathrm{Se}$ reaction. Over the experimental data the comparisons are very good, typically within $1-5 \%$. For additional comparisons, see (Koning \& Delaroche 2001).

\subsubsection{Transmission Coefficients for Photons}

For the calculation of the gamma ray transmission coefficients, we use a simple model where the transmission coefficient depends only on the multipole type (XL) and the transition energy $(\epsilon)$. They are related to the gamma ray strength function $f_{X L}^{\gamma}(\epsilon)$ by

$$
T_{X L}^{\gamma}(\epsilon)=2 \pi \epsilon^{2 L+1} f_{X L}^{\gamma}(\epsilon)
$$

The energy dependence of the strength function was determined using the GDR model with Lorentz line shapes. The transmission coefficients
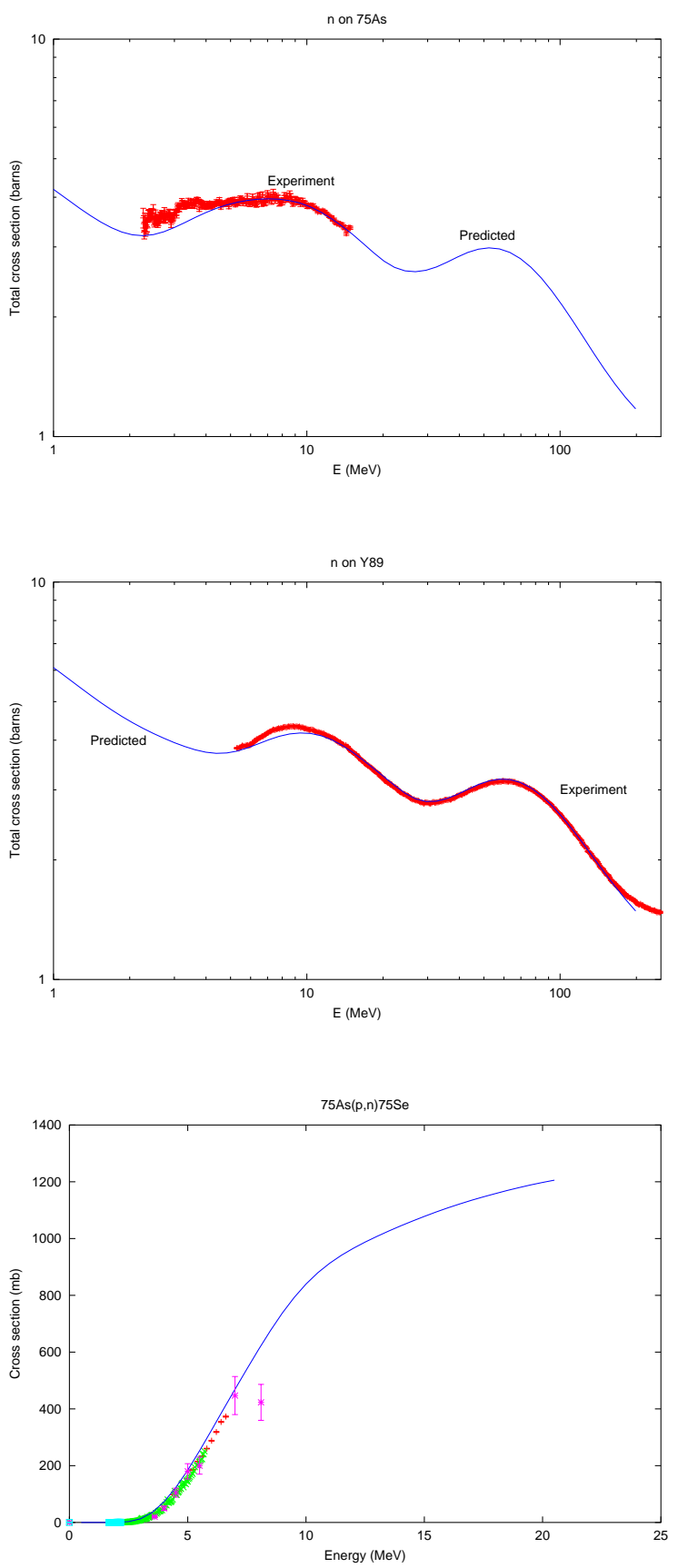

Fig. 1.- Total neutron and proton Cross Sections vs Koning-Delaroche

were then normalized by fitting the average total s-wave radiation width at the neutron binding energy (Uhl \& Strohmaier 1976). 
Since the total s-wave radiation width at the neutron binding energy is generally measured only for stable isotopes plus a neutron, we developed a systematic approach for estimating this value for the many unstable nuclei in our region of interest. The systematic values are determined by a least squares linear fit to experimental data, with separate systematics developed for even-Z even-N, even-Z odd-N, odd-Z even N, and odd-Z odd-N nuclei. In the case of odd- $Z$ even- $N$ nuclei, no experimental s-wave data was available in our region of interest, so we adopted the odd-Z odd-N systematic for all odd-Z nuclei (Figure 2). Of course we used the experimental values for all systems that had measured average s-wave radiation widths.

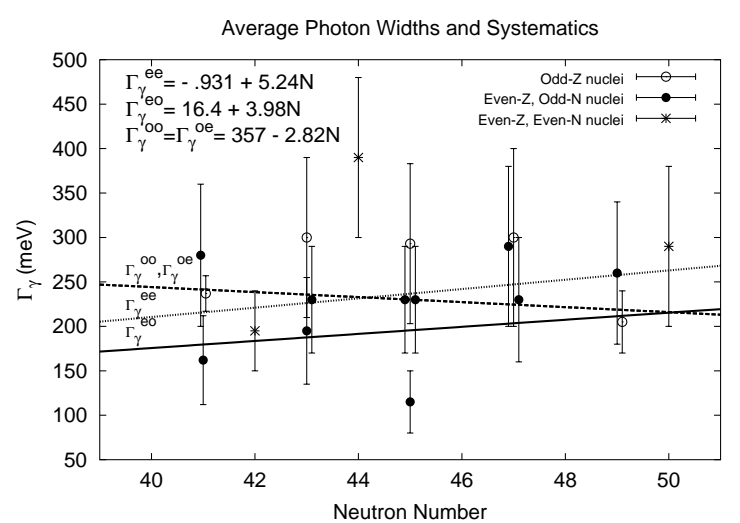

Fig. 2.- Systematics for average total s-wave radiation width.

\subsection{Nuclear Level Densities}

\subsubsection{Level Density Models}

Possibly the most important input to the statistical model code, especially for the capture reactions, is the nuclear level density. For this project, we have adopted a standardized, semi-empirical approach (Gilbert \& Cameron 1965) which is numerically efficient, can be tied to experimental data, and is fairly accurate. The level density is described by two functions. Both are energy dependent, the second factor contains the spin dependence. This is the "Back-shifted Fermi Gas" formulation of the nuclear level density:

$$
\rho(U, J)=\rho(U) f(U, J)
$$

where $\rho(U)$ is the state density, with $U=E-\Delta$ the back-shifted energy. $\Delta$ is the so called "pairing energy", and $J$ is the spin of the compound nucleus. We will further treat each of these in two ways, depending on the excitation energy of interest. The demarcation point will be roughly between the energy range of the known excited levels of a given compound nucleus (the low energy domain), and near (and above) the neutron binding energy (the high energy domain).

For the high energy domain, we describe the level density assuming a Fermi gas formula,

$$
\begin{gathered}
\rho(U)=\frac{\sqrt{\pi}}{12} \frac{\exp (2 \sqrt{a U})}{a^{1 / 4} U^{5 / 4}} \frac{1}{\sqrt{2 \pi} \sigma} \\
f(U, J)=\frac{2 J+1}{2 \sigma^{2}} \exp \left[\frac{-\left(J+\frac{1}{2}\right)^{2}}{2 \sigma^{2}}\right]
\end{gathered}
$$

where $a(E)$ is the level density parameter (in $\left.\mathrm{MeV}^{-1}\right)$. The spin cutoff parameter $\sigma^{2}$ is defined as

$$
\sigma^{2}=\lambda \sqrt{a U} A^{2 / 3}
$$

The level density assumes an equal distribution of parity states. Note that at low excitation energy (for a positive back-shift), Eq. [5] diverges. At low energies, the nuclear level density is better described by a constant temperature formula:

$$
\rho(E) \propto \exp \frac{E-E_{0}}{T}
$$

The level density parameters can be calculated using experimental data. For the Fermi-gas state density (Eq. [5]), the level density parameter, $a(E)$, can be related to the average level spacing $\left(D_{0}\right)$ near the neutron binding energy. The pairing energies used in the calculation of the back shifted energy are calculated as differences of binding energies (Bohr \& Mottelson ). The constant temperature parameters $E_{0}$ and $T$, can be chosen to provide a state density that goes through the low lying spectroscopic levels subject to the choice of a matching energy, $E_{x}$, chosen someplace between the high and low energy regions of interest, at which the two state densities match (point and slope). We describe below how we determined these parameters for all of the nuclei considered in this study. 


\subsubsection{Level Densities Above the Neutron Bind- ing Energy}

Our goal is to fit the level density parameter $a$ in Eq. [5] to experimental data where available. We adopt an energy dependent form, $a(U, Z, N)$ (Iljinov et al. 1992), and begin by fixing the spin cutoff parameter and the pairing energies.

\section{The Spin Cutoff Parameter}

The spin cutoff parameter $\sigma^{2}$, Eq. [7], characterizes the spin distribution of the fermi gas level density. It depends on the parameters $a$, the level density parameter, and $\lambda$, which determines the effective moment of inertia for the nucleus in question. In principle it could be determined by experiment, for example, by comparing ratios of cross sections leading to different isomers of the product nucleus (Keisch 1963). Because data like this is often sparse, especially in the limited regions of the periodic chart we are interested in, and because we are often interested in reactions that proceed on or through radioactive species where no such data exists, we must resort to models. In our analysis, we chose to fix $\lambda=0.124$ in Eq. [7]. This is a reasonable average of the values proposed by Gilbert and Cameron (0.0888) (Gilbert \& Cameron 1965) and Rauscher (0.146) (Rauscher et al. 1997).

\section{Pairing Energies}

In determining the back-shift $\Delta$, also known as the pairing energy, we used a slightly modified version of the method of Rauscher (Rauscher et al. 1997). The total pairing energy is equal to the sum of the proton and neutron pairing energies

$$
\begin{aligned}
\Delta(Z, N) & =\Delta_{p}+\Delta_{n} \\
\Delta_{p}(Z, N) & =E^{G}(Z, N) \\
& -\frac{1}{2} E^{G}(Z-1, N) \\
& -\frac{1}{2} E^{G}(Z+1, N) \\
\Delta_{n}(Z, N) & =E^{G}(Z, N) \\
& -\frac{1}{2} E^{G}(Z, N-1) \\
& -\frac{1}{2} E^{G}(Z, N+1)
\end{aligned}
$$

where $E^{G}(Z, N)$ is the binding energy of the nucleus $(Z, N)$. In calculating the binding energies of the various nuclei, we used the experimental mass excesses listed in the Möller and Nix tables (Möller et al. 1995).

\section{The Level Density Parameter}

At high energies, the level density parameter $a$ behaves essentially as a function of mass number only. However, in cases where deformation and shell effects are important (often the case near closed neutron shells) it has been shown (Iljinov et al. 1992) that at low energies it is more appropriate to use an energy dependent form of the level density parameter;

$$
a(U, Z, N)=\tilde{a}(A)\left[1+\delta W(Z, N) \frac{f(U)}{U}\right]
$$

with

$$
f(U)=1-\exp (-\gamma U)
$$

and as usual $U=E-\Delta$. In previous works where a more global prescription was developed [(Rauscher et al. 1997), (Iljinov et al. 1992)], one would adopt a semi-empirical shell correction, $\delta W(Z, N)$, and fit $\tilde{a}(A)$ to known experimental data. Here we choose to adopt a simple form for the mass dependent term and fit the shell correction.

In our analysis, we followed the convention of (Rauscher et al. 1997) in choosing the parameter $\gamma=0.04884$ that describes the fall off in energy of the shell correction. We also chose a relatively simple form for the asymptotic value of the level density parameter, $\tilde{a}(A)=0.125 \times A=A / 8$ (the classical result). It was discovered that in the energy range of interest to us, the exact form of $\tilde{a}(A)$ made very little difference in determining the shell corrections $\delta W$ from experimental values of resonance spacings.

\section{Shell Corrections}

Shell corrections can be determined for select nuclei from experimental values of the average level spacings $D_{0}$ as determined by neutron resonance analysis (RIPL 1998). For $s$-resonances (neutron angular momentum equal to zero), the calculated level spacing, $D_{\text {calc }}$, evaluated at the neutron 
binding energy $U=B_{n}$, is related to the nuclear level density (e.g. Eq.'s [4-6])

$$
D_{\text {calc }}=\frac{2}{\rho\left(U, J=\frac{1}{2}\right)}
$$

for nuclei with $s=0$ and

$$
D_{\text {calc }}=\frac{2}{\rho\left(U, J=s+\frac{1}{2}\right)+\rho\left(U, J=s-\frac{1}{2}\right)}
$$

for nuclei with $s \neq 0$. In each case, the level densities $\rho(U, J)$ were calculated using the other parameters $(\lambda, \Delta, \gamma, \tilde{a}(A))$ set as described previously. We then numerically solved for the value of $\delta W$ that would minimize the quantity $D_{\text {calc }}-D_{0}$ using root bisectioning methods. This now completely describes the parameterization of $\sigma^{2}$ and $a(U, Z, N)$, and thus the Fermi-gas level density.

\subsubsection{Systematic Behavior of Fermi Gas Level Density Parameters}

There are only a limited number of nuclei for which the average resonance spacing $D_{0}$ has been measured (i.e. for compound nuclei formed from a stable target plus a neutron). As a result, we were required to systematically predict the shell correction for the remaining nuclei in the range of interest for this project. After plotting the experimental $\delta W$ in our region of interest and their associated errors and adding $2 \mathrm{MeV}$ to the odd- $\mathrm{Z}$ nuclei, we noted a roughly linear behavior. We made a $\chi^{2}$ linear fit to the data, shown in figure 3. We then subtracted $2 \mathrm{MeV}$ from the $\delta W$ for odd-Z nuclei to account for the $2 \mathrm{MeV}$ added prior to making the systematic. Of course, where available, we always used an experimentally determined shell correction over a systematic one.

\subsubsection{Level Densities Below the Neutron Bind- ing Energy}

For the lower energy regions, below the neutron binding energy $B_{n}$, the nuclear level density has the same formulation as Eq. [(4)]. However, particularly at and below the pairing energy $\Delta$, the state density in Eq. [5] becomes imaginary. Unfortunately, experimental level schemes are rarely known above $2 \mathrm{MeV}$ of excitation energy. In practice we are forced again to assume a model and use all available experimental data to constrain its parameters.

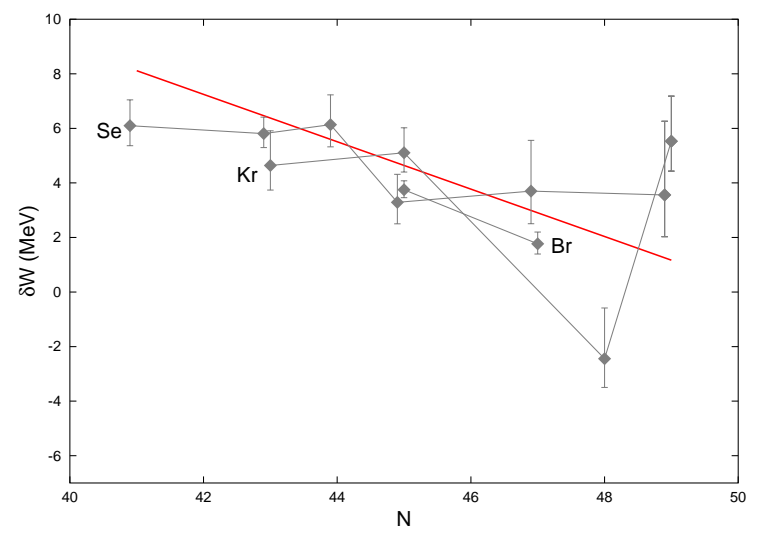

Fig. 3.- $\chi^{2}$ linear fit to experimentally determined shell corrections, used to systematically determine unknown shell corrections.

Of course the two prescriptions for the level density must match at some energy intermediate to where they are constrained by experiment. Henceforth we will refer to the high energy level density as $\rho_{1}$, and the low energy density as $\rho_{2}$.

Gilbert and Cameron (Gilbert \& Cameron 1965) noticed that the cumulative number of observed levels (the so-called staircase plot, which increase exponentially), can be fit with straight lines in a semi-log plot. They adopted a constant temperature formula to fit these:

$$
N(E)=\exp \left[\frac{E-E_{0}}{T}\right]
$$

with $N(E)$ being the cumulative number of levels at excitation energy $E, E_{0}$ and $T$ are two free parameters to be fit to the observed level structure. The observable level density is given by

$$
\rho_{1}(E)=\frac{d N(E)}{d E}=\frac{1}{T} \exp \left[\frac{E-E_{0}}{T}\right]
$$

From classical thermodynamics, we have a definition of the nuclear temperature

$$
\frac{d}{d E} \log \rho_{1}(E)=\frac{1}{T}
$$

where $T$ now takes on the meaning of a nuclear temperature which is constant in the region of the discrete levels. We assume that Eq. [14] can be extrapolated from the region of the known discrete 
levels to higher energies, where the fermi-gas level density $\left(\rho_{1}\right)$ is valid. We then define the notion of a fit to the total level density over the entire range as being achieved if: a) a good fit can be made to the low lying levels, b) the observed level spacing at the neutron binding energy is exactly reproduced, and c) the energy of the matching point $E_{x}$ for the two prescriptions falls between $E=0$ and $E=$ $B_{n}$, and that they match at this point with the same slope, i.e. for $E=E_{x}$ :

$$
\begin{aligned}
\rho_{1}\left(E_{x}\right) & =\rho_{2}\left(E_{x}\right) \\
\frac{d \log \rho_{1}\left(E_{x}\right)}{d E} & =\frac{d \log \rho_{2}\left(E_{x}\right)}{d E}
\end{aligned}
$$

From the first of these, we can determine $E_{0}$ :

$$
E_{0}=E_{x}-T \log T \rho_{2}\left(U_{x}\right)
$$

where $U_{x}=E_{x}-\Delta$. The second condition can be satisfied by assuming that at $E_{x}$ the constant nuclear temperature $T$ of the low lying states is equal to the energy dependent nuclear temperature $\tau\left(U_{x}\right)$ of the high excited states,

$$
\begin{aligned}
\frac{1}{T} & =\sqrt{\frac{a}{U_{x}}}-\frac{3}{2 U_{x}} \\
& +\frac{(\tilde{a}-a)\left(1+\gamma U_{x}\right)+\tilde{a} \gamma \delta W}{\sqrt{a U_{x}}}
\end{aligned}
$$

where $a$ is given by Eq. [10]. If there is no shell correction, the latter term in the above equation is zero. Typical values for the matching energy are $2 \leq E_{x} \leq 5 \mathrm{MeV}$, and are approximated by $E_{x}=2.5+\frac{150}{A}+\Delta$ (Gilbert \& Cameron 1965). An example of a fit to the low lying levels of ${ }^{79} \mathrm{Br}$ is presented in Figure (4).

\section{Behavior of the Spin Cutoff Parameter Below $E_{x}$}

At the matching energy $E_{x}$, the spin cutoff parameter is given by Eq. [7]. Below this, we treat the behavior of the spin cutoff parameter differently.

We define $E_{\text {cut }}$ as the energy of the highest known excited level for which energy, spin and parity are explicitly known. Using the known spectroscopic levels, we define a low energy spin cutoff parameter at $E_{\text {cut }}$ :

$$
\sigma_{E_{c u t}}^{2}=\frac{1}{2 N} \sum_{1}^{N}\left(J_{i}+\frac{1}{2}\right)^{2}
$$

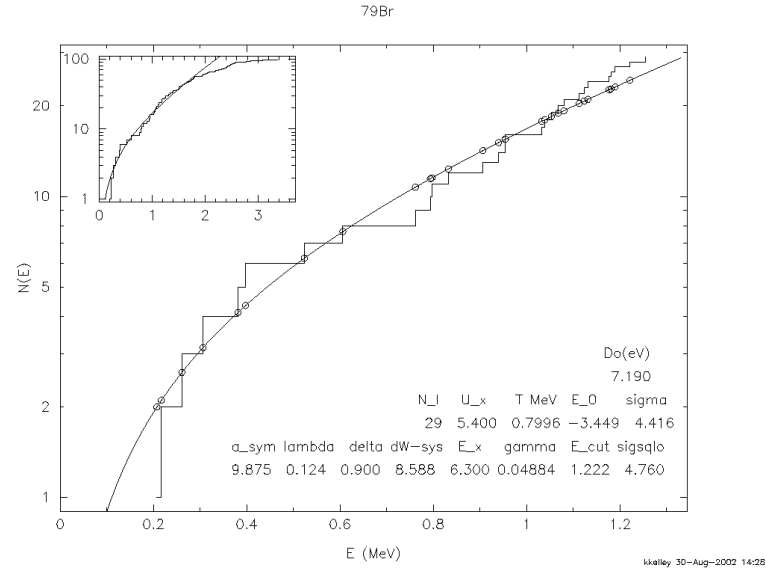

Fig. 4. - Sample of a typical fit to low lying spectroscopic levels

where $\mathrm{N}$ represents the number of the level with energy $E_{c u t}, J_{i}$ are the spins of the individual levels, and we exclude the ground state $(\mathrm{i}=0)$. This value is used for energies $0 \leq E \leq E_{\text {cut }}$.

For energies $E_{\text {cut }} \leq E \leq E_{x}$, the spin cutoff parameter is given by a linear fit between these two values (Eq.'s [7] and [21]). Specifically,

$$
\sigma_{I}^{2}=\sigma_{E_{x}}^{2}-\frac{\sigma_{E_{x}}^{2}-\sigma_{E_{c u t}}^{2}}{E_{x}-E_{c u t}}\left(E_{x}-E\right)
$$

This conforms to the treatment of $\sigma^{2}$ in the IDA reaction code system (Reffo 1978).

Another treatment, used in the GNASH code system (Chadwick 1998), defines:

$$
\begin{aligned}
\sigma_{H}^{2} & =\sigma_{E_{x}}^{2} \\
U_{L} & =\max \left(E_{c u t}-\Delta, 0.1\right) \\
\sigma_{L}^{2} & =\lambda \sqrt{a U_{L}} A^{2 / 3} \\
\sigma_{G}^{2} & =\sigma_{L}^{2}+\frac{E-\frac{1}{2} E_{c u t}}{E_{x}-\frac{1}{2} E_{c u t}}\left(\sigma_{H}^{2}-\sigma_{L}^{2}\right)
\end{aligned}
$$

The fitted parameters for the total level density are presented in Table 5. The symbols in the legend are the same as described above. In column five, an " $\mathrm{x}$ " indicates the shell correction $\delta W$ was derived from an experimentally known level spacing $D_{0}$, an "s" indicates the shell correction was derived from the systematic shown in Figure 3. In column 10, an "I" indicates the spin cutoff parameter below the matching energy $E_{x}$ was treated as 
in Eq. [22], while a "G" indicates the GNASH treatment was used (Eq. [23]).

\section{Calculated Cross Sections}

\subsection{Comparison to Measured Cross Sec- tions}

Having developed the various input quantities based on available experimental data in the previous section, we now turn to results of the STAPRE-H95 model and compare to available experimentally measured cross sections in the region of interest. Only results for targets initially in their ground states are available.

In Figure (5) we present the comparison for $(\mathrm{n}, \gamma),(\mathrm{n}, 2 \mathrm{n})$, and $(\mathrm{p}, \mathrm{n})$ reactions on the target ${ }^{79} \mathrm{Br}$. These comprise the main destruction reactions for the principle loaded detector element for bromine. Shown is the activation cross section (solid black lines in all plots that follow) defined as the sum of emission (both particle emission and gamma-ray cascade) from the compound nucleus that eventually leads to the ground state of the product (final) nucleus. We also provide (where appropriate) separate cross sections that decay to the ground state (red lines), and any long lived isomer (blue lines, see Table 2 for a list of the isomers and their respective lifetimes). These cross sections are plotted against the available experimental data, taken from the Experimental Nuclear Reaction Data File (CSISRS 2003). Cross sections for the total, ground, and isomeric states are colored the same as the modeled cross sections (black is activation, red is to ground, and blue to an isomer, respectively), with different symbols distinguishing results from various experiments.

For ${ }^{79} \mathrm{Br}(\mathrm{n}, \gamma)^{80} \mathrm{Br}$, our result for all three cross sections (a, g, m1) are slightly low. At roughly 200 $\mathrm{keV}$ the activation cross section is lower by $20 \%$, but at $25 \mathrm{keV}$ the difference is only $10 \%$. This is a very good result for an $(\mathrm{n}, \gamma)$ activation cross section. Even better agreement was obtained for ${ }^{81} \mathrm{Br}(\mathrm{n}, \gamma){ }^{82} \mathrm{Br}$. The results for the ground state are better $(\sim 5 \%$ between 10 and $100 \mathrm{keV})$, but the isomer cross section is low by roughly 25activation cross section that is used to infer activity in the UGT program. Using global systematics, (n, $\gamma)$ cross sections can typically be modeled within a factor of two, often to within $30 \%$ (Hoffman et al. 1999).
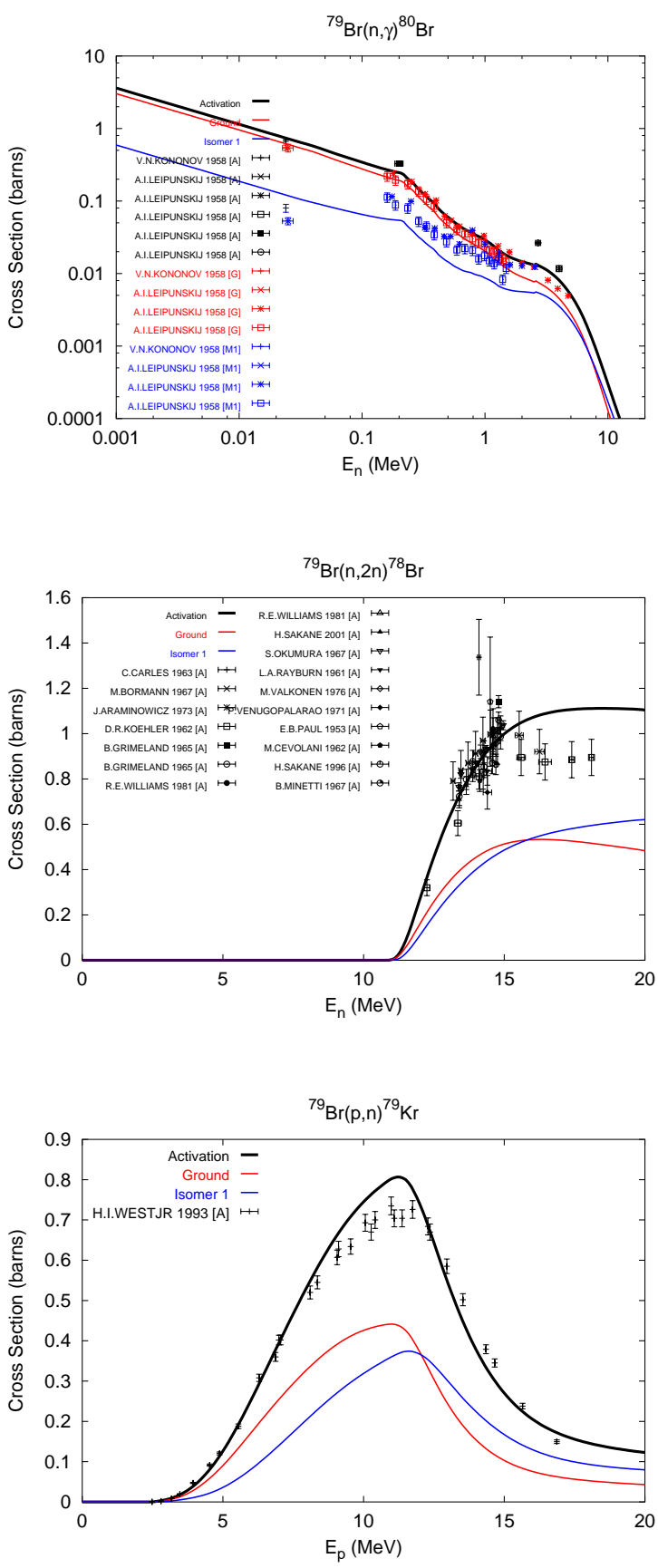

Fig. 5.- Calculated vs. Experimental Cross Sections: ${ }^{79} \mathrm{Br}$.

The ${ }^{79} \mathrm{Br}(\mathrm{n}, 2 \mathrm{n})^{78} \mathrm{Br}$ activation cross section shows good agreement with numerous experimental efforts around $14 \mathrm{MeV}$, although the spread 
among them is rather large (about 25\%). The most recent measurements seem to be in the best agreement. Predicting $(\mathrm{n}, 2 \mathrm{n})$ cross sections is fairly straightforward, as they scale roughly with the size of the nucleus, the activation cross section is typically about a barn at $14 \mathrm{MeV}$ regardless of atomic number.

Finally, the ${ }^{79} \mathrm{Br}(\mathrm{p}, \mathrm{n}){ }^{79} \mathrm{Kr}$ cross section shows a very good shape over the entire excitation function, but our calculated value is slightly higher than that measured by (West et al. 1993). The agreement for ${ }^{81} \mathrm{Br}(\mathrm{p}, \mathrm{n}){ }^{81} \mathrm{Kr}$ is in slightly better agreement at its peak. For purposes of RADCHEM analysis, we use the measured cross sections in place of these modeled ones, although these results should be considered as very reasonable.

In our region of interest there are 18 other cross sections for which experimental data is available. These are shown in Appendix A.6. Figure (20) shows (n, $\gamma$ ) cross sections that exhibit similar agreement (within $\sim 20 \%$ ) to the bromine capture reactions. The situation for ${ }^{80} \mathrm{Se}(\mathrm{n}, \gamma){ }^{81} \mathrm{Se}$ is difficult to gauge due to a large spread in the experimental data. We note that our calculation at low energies $(\sim 25 \mathrm{keV})$ may suffer from having only 5 experimental levels included in the gammacascade for ${ }^{81} \mathrm{Se}$ (see Appendix A.4). This will affect the isomer cross section in particular. Overall we believe we have well predicted the measured $(\mathrm{n}, \gamma)$ cross sections in the entire region of interest, and we expect our calculated capture cross sections on ${ }^{79,81} \mathrm{Kr}$ to have similar accuracy.

Yet another comparison to experimental data comes from the extensive efforts to evaluate maxwellian averaged capture cross sections for astrophysical applications (Bao et al. 2000). The Maxwellian-averaged neutron capture cross section is defined as the reaction rate $\langle\sigma v\rangle$ divided by the mean velocity $v_{T}=\sqrt{2 k T / \mu}$ at a given temperature $T$. Here, $\mu$ is the reduced mass. For particle fluences and temperatures typical to stellar nucleosynthesis, the velocity distribution of the neutrons reduces to a Maxwell-Boltzmann distribution. In this case, the Maxwellian-averaged cross section reduces to (Beer et al. 1992)

$$
\frac{\langle\sigma v\rangle}{v_{T}}=\frac{\int_{0}^{\infty} \sigma_{n \gamma} v \Phi(v) d v}{v_{T}}
$$

$$
=\frac{2}{\sqrt{\pi}(k T)^{2}} \int_{0}^{\infty} \sigma_{n \gamma}(E) W(E, k T) d E
$$

where $W(E, k T)=E \exp (-E / k T)$ and $E$ is the center of mass energy.

Figure 21 comapres our calculated Maxwellianaveraged capture cross sections for ${ }^{76,78} \mathrm{Se},{ }^{79,81} \mathrm{Br}$, and ${ }^{78,80} \mathrm{Kr}$ to the evaluated cross sections from (Bao et al. 2000). The error bars on all points are identical and represent the measured error for a given cross section at $30 \mathrm{keV}$. We used spline interpolation to determine the value of the $(\mathrm{n}, \gamma)$ cross section between points on the energy grid. For energies below our lowest grid energy, we assume an $(\mathrm{n}, \gamma)$ cross section with an $E_{l a b}^{-1 / 2}$ dependence. For energies greater than our highest grid energy, we take the cross section to be zero.

Overall our calculated cross sections compare very favorably to those of (Bao et al. 2000), especially for the two Bromine targets. Again we are well within $30 \%$ accuracy over the mass and energy range of interest here, often better, consistent with the comparisons shown in Figure 20.

Figure (22) shows (n,2n) cross sections that exhibits overall good agreement (within $\sim 10 \%$ ), similar to the bromine $(\mathrm{n}, 2 \mathrm{n})$ activation cross sections. Even the prediction of the relative ground to isomer $(\mathrm{n}, 2 \mathrm{n})$ cross sections on ${ }^{80} \mathrm{Se}$ and ${ }^{79} \mathrm{Br}$ exhibit good agreement (although the reported activation cross section of Okumura is in serious disagreement, it seems more in line with the other results for the first isomer cross section). The agreement for the even- $\mathrm{Z}$, even- $\mathrm{N}$ targets is uniformly good. We expect the $(\mathrm{n}, 2 \mathrm{n})$ cross sections on odd- $\mathrm{N}$ targets to be accurate to about $\sim 10 \%$.

Finally, Figure (23) shows $(\mathrm{n}, \mathrm{x})$ cross sections. The experimental data is often sparse and over a limited energy range, but the agreement is still rather good. This includes the destruction $(\mathrm{n}, \mathrm{p})$ cross sections on ${ }^{80,82} \mathrm{Kr}$. We do note that $(\mathrm{n}, \mathrm{p})$ cross sections on even- $\mathrm{N}$ targets are small $(\sim 100$ $\mathrm{mb}$ ), although they are large on odd- $\mathrm{N}$ targets $\left({ }^{77,79} \mathrm{Kr}\right.$, see Appendix A.7).

\subsection{Sensitivity Studies}

We illustrate the sensitivity of our modeled results to variations in the input parameters developed in $\S 3$ against the measured activation cross sections for $(\mathrm{n}, \gamma),(\mathrm{n}, 2 \mathrm{n})$, and $(\mathrm{p}, \mathrm{n})$ reactions on ${ }^{79} \mathrm{Br}$. 


\subsubsection{Sensitivity to the Pre-Equilibrium Cross Section}

We adopt a simple exciton model with initial 2-particle 1-hole configuration. Average rates for internal transitions are related by the formulas of Williams (1970), corrected for the Pauli principle by Cline (1972), to the absolute square of the average effective matrix element $|M|$ of the residual interactions as per Eq. (7) of (Uhl \& Strohmaier 1976). The dependence of $|M|^{2}$ on mass number and excitation energy is

$$
|M|^{2}=\langle F M\rangle A^{-3} E^{-1}
$$

Figure (6) shows the sensitivity of the ${ }^{79} \operatorname{Br}(\mathrm{p}, \mathrm{n})$ cross section for variations of $50 \leq\langle F M\rangle \leq 200$. The variations affect the high energy tail of the cross section, with a value of $\langle F M\rangle=100$ providing reasonable agreement with the experimental excitation function. This value for $\langle F M\rangle$ was applied in calculating the pre-equilibrium contribution to all cross sections in this study.

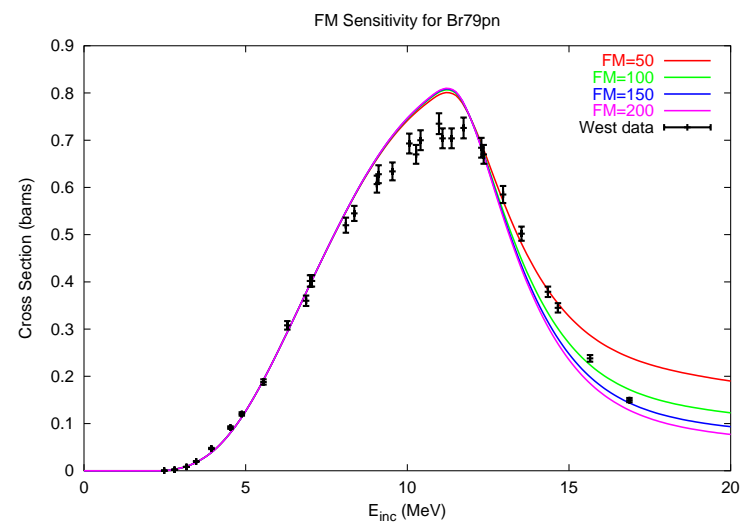

Fig. 6.- Sensitivity to Pre-Equilibrium Matrix Element.

\subsubsection{Sensitivity to the choice of Level Density Prescription}

The nuclear level density parameters developed for this region (Table 5) reflect best choices from the available experimental data in the region of interest. Instead of varying each of the many parameters $\left(a(U, Z, N), \sigma^{2}, \lambda, \Delta, \delta_{W}\right)$, we instead present results where only the overall treatment of the level density prescription was varied, keeping all other parameter input fixed.
Figure (7) shows the experimental cross sections for $(\mathrm{n}, \gamma),(\mathrm{n}, 2 \mathrm{n})$, and $(\mathrm{p}, \mathrm{n})$ cross sections on ${ }^{79} \mathrm{Br}$ (for the sources of the data, see Figure 5). For comparison we show our local systematic level density (red-solid line, Table 5).

The second level density prescription from (Rauscher et al. 1997) is plotted as the purple dashed line. This prescription is the current standard used in the calculation of cross sections for use in stellar nucleosynthesis calculations (Rauscher et al. 2002). Rauschers prescription is similar to ours, in that it also embodies an energy dependent $a(E)$ parameter (e.g. (Iljinov et al. 1992)) with shell corrections that damp out with increasing excitation energy (see our Eq. 10).

A third level density prescription is shown as the blue dotted line (Gilbert \& Cameron 1965). This is one of the original prescriptions available and has been used to model some of the cross sections in the current Br-Kr RADCHEM sets (Vonach 1982). It includes pairing and shell corrections but with an energy independent $a(A)$ parameter. It was also the prescription used in the first large scale theoretical cross section library developed for use in modeling pre-supernova nucleosynthesis in stars[(Woosley et al. 1978), (Woosley \& Weaver 1995)], and provided remarkable success in predicting measured solar abundances within the context of a study of Galactic Chemical Evolution (i.e. radchem for astrophysics). See (Timmes Woosley \& Weaver 1995).

The last choice reflects a recent attempt to calculate level densities using microscopic nuclear structure models (Goriely 2002).

For the $(\mathrm{n}, \gamma)$ capture reaction, we see that three of the prescription's (ours, GC, and Goriely) all predict the same cross section below $1 \mathrm{MeV}$, and in good agreement with the experimental data below $200 \mathrm{keV}$. Rauscher appears to be low throughout. All prescriptions indicate slightly different behavior above $1 \mathrm{MeV}$, with the Goriely treatment appearing to agree best with the high energy points near $4 \mathrm{MeV}$. Of course above $1 \mathrm{MeV}$ the value of the cross section begins to drop rapidly $(\leq 10 \mathrm{mb})$, and so this would not compete with particle induced reactions for these higher energies. Overall, we believe our local systematic is in good agreement for the important energy range between 10 and $100 \mathrm{keV}$. 

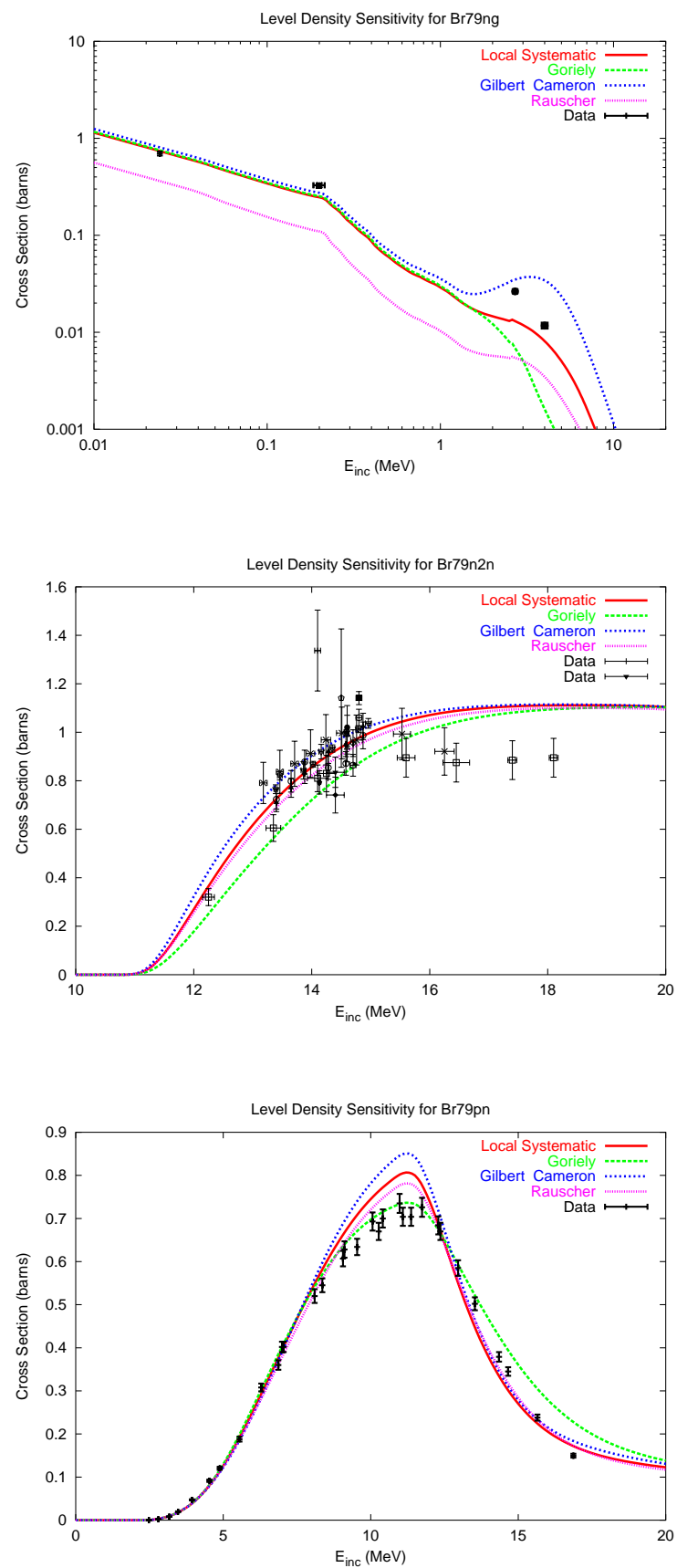

Fig. 7.- Sensitivity to the Level Density Prescription.

For the $(n, 2 n)$ reaction we see that the three Fermi-Gas level densities all predict roughly the same cross section (with variations of order $\sim 5 \%$ ) over the entire range of excitation energy studied. The microscopic level density of Goriely is lower by roughly $30 \%$ near $14 \mathrm{MeV}$. Again, we consider our local systematic to be preferable to any of the other three.

Finally, for the $(p, n)$ cross section we see the choice of level density only affects the peak of the $(p, n)$ cross section, with some difference in shape for the high energy tail exhibited by the Goriely treatment, who coincidentally appears to best replicate the peak in the experimental data. The variation at peak is roughly $12 \%$, about twice the uncertainty in the experimental data. Again, we consider our local systematic to do a good job on this cross section, recalling that we do use the actual measured cross section (West et al. 1993) in our RADCHEM calculations.

\subsubsection{Sensitivity to the Normalization of the $\gamma$ - ray Transmission Coefficient}

Figure 8 shows the sensitivity to a $\pm 30 \%$ change to the value of the average s-wave photon width used to normalize the gamma-ray transmission coefficients. The $30 \%$ variation translates into a nearly identical change in the cross section from 10 $\mathrm{keV}$ to $1 \mathrm{MeV}$. The $(\mathrm{n}, 2 \mathrm{n})$ and $(\mathrm{p}, \mathrm{n})$ cross sections do not exhibit any sensitivity because $\mathrm{T}_{\gamma}$ only entered into Eq. [1] in the denominator. In general, the smaller of the two transmission coefficients in th HF numerator will be the one that determines the cross section, especially if it is much smaller. This is always the case with photon vs. particle widths.

\subsubsection{Sensitivity to the Width Fluctuation Cor- rection}

We adopt the Moldauer model of the WFC as embodied in the STAPRE code (Uhl \& Strohmaier 1976). For targets in the mass range of $\mathrm{Br}$ and $\mathrm{Kr}$ which have available experimental data, reaction thresholds are always greater than several $\mathrm{MeV}$, and width fluctuation corrections will only be evident for capture reactions. Figure 9 shows the affect for the activation capture cross section of ${ }^{81} \mathrm{Br}$ both with (solid black line) and without (red line) WFC. As expected an decrease in the capture cross section is noticable, although small (a few percent), below $500 \mathrm{keV}$. When the projectile energy increases, the capture cross section 


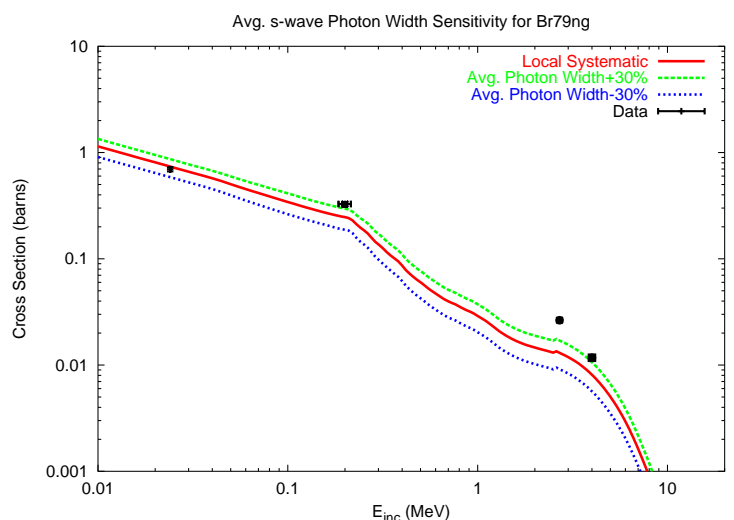

Fig. 8. - Sensitivity to the s-wave Average Photon Width.

delcines rapidly and the eleastic enhancement vanishes. The $(n, p)$ and $(p, n)$ channels do not open until the incident neutron energy is above 2 $\mathrm{MeV}$, and so no other channels can compete with the elastic and capture cross section before the WFC are negligable. Nearly identical behaviour is noted for neutron catpure on ${ }^{88} \mathrm{Sr}$ and ${ }^{93} \mathrm{Nb}$ (Hilaire Lagrange \& Koning 2003), see their Figures 2 and 3 .

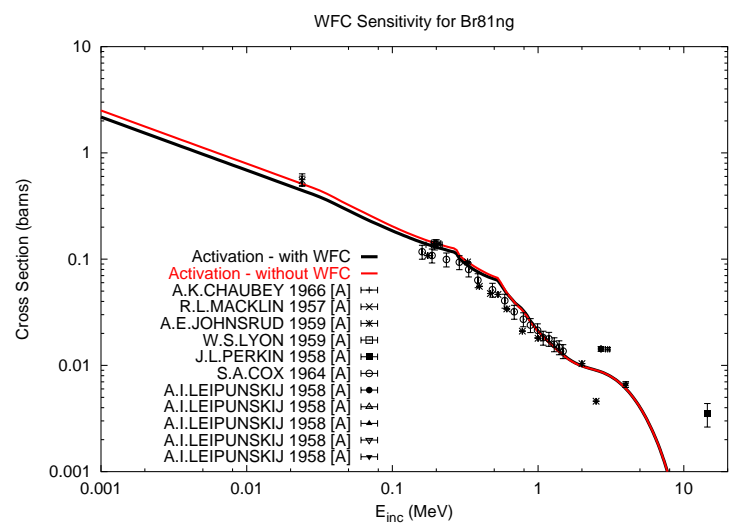

Fig. 9.- Sensitivity to the Width Fluctuation Correction.

\subsection{Production and Destruction Cross Sections}

Figure 10 shows the modeled cross sections that directly affect the production and destruction of ${ }^{79} \mathrm{Kr}$. Identical plots for the other targets are presented in the Appendix (Figures 24-30).

The modeled $(\mathrm{p}, \mathrm{n})$ production cross section is the only one of importance, since ${ }^{79} \mathrm{Br}$ is the loaded isotope $(50.69 \%)$. Secondary pathways exist that proceed from ${ }^{81} \mathrm{Br}$, but these would require population of the intermediate radioactive isotopes ${ }^{80} \mathrm{Br}$ and ${ }^{80} \mathrm{Kr}$. The largest cross section below $5 \mathrm{MeV},{ }^{79} \mathrm{Rb}(\mathrm{n}, \mathrm{p}){ }^{79} \mathrm{Kr}$, is not important, as this would require successive $(\mathrm{p}, \mathrm{n})$ reactions on the loaded species with a subsequent $(\mathrm{n}, \mathrm{p})$ reaction to bringing material back to ${ }^{79} \mathrm{Kr}$. Of course the particle fluenences play a dominant role in determining the most important cross sections. More specific details will be disclosed in the classified analysis presented in a following paper.

Of the destruction cross sections, the largest is

${ }^{79} \mathrm{Kr}(\mathrm{n}, \gamma)$, becoming significant below $2 \mathrm{MeV}$ of excitation energy. There is competition at intermediate energies between 1 and $8 \mathrm{MeV}$ due to the $(\mathrm{p}, \mathrm{n})$ and $(\mathrm{n}, \mathrm{p})$ reactions on ${ }^{79} \mathrm{Kr}$. It should be noted that $(\mathrm{n}, \mathrm{p})$ cross sections were not included in either of the previous sets (Br0391 and Kr0991), and no reactions on $\mathrm{Rb}$ were included. We consider these to be unlikely in affecting the overall production of ${ }^{79} \mathrm{Kr}$.

\subsubsection{Results for Remaining Reactions in the Detector Set}

Figures 24-30 show all modeled cross sections for the elements $\mathrm{Se}, \mathrm{Br}$, and $\mathrm{Kr}$ arranged by increasing neutron number. The variations on each cross section are often small, with the exception of the $(\mathrm{n}, \mathrm{p})$ cross sections. For the lower neutron numbers $Q_{n p}$ becomes exoergic (Table 4), and the low energy behavior starts to assume a $1 / \sqrt{(} E)$ behavior, as does $(\mathrm{n}, \gamma)$. In this mass range the affects of $(n, p)$ cross sections are beginning to become important; for lower masses, around Ti and $\mathrm{Cr}$, they will play a larger role as the dominant destruction cross sections. 

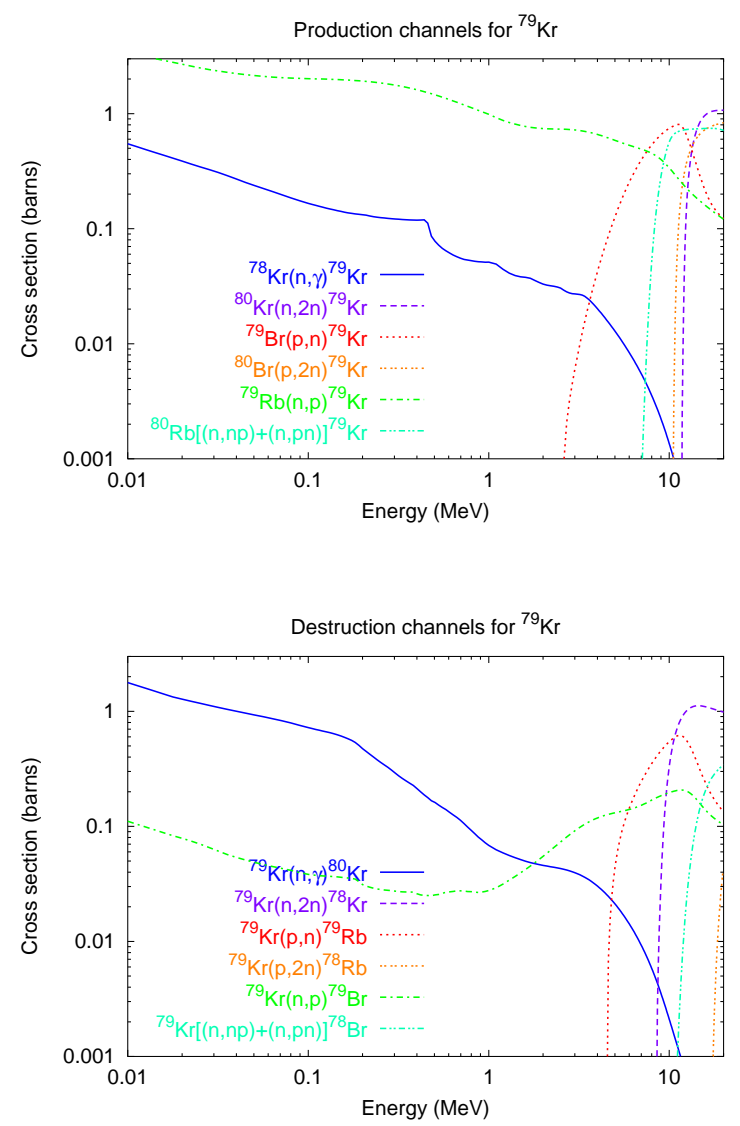

Fig. 10.- Calculated Cross Sections directly affecting production and destruction of ${ }^{79} \mathrm{Kr}$.

\section{Conclusions}

We have developed a new charged particle cross section set for radiochemical diagnostics. The theory and implementation of the Hauser-Feshbach model were described, along with the details of the local systematics used to create a set of input parameters that reflect the latest available experimental data in the region of interest around ${ }^{79} \mathrm{Br}$. Sensitivity to the input models and parameters was explored. The choice of our developed local systematics appears to well replicate measured cross sections over this restricted region of interest. Since we are interested here in the production of one radioactive species, ${ }^{79} \mathrm{Kr}$, the number of reactions that can play a role in its activation are small. A similar case exists for another RADCHEM charged particle detector, ${ }^{127} \mathrm{I}$ pro- ducing ${ }^{127} \mathrm{Xe}$, also a noble gas. This will be the next set to be published and we anticipate similar success in our modeling effort when comparing to the many measured cross sections in that area of the periodic chart.

Overall we consider the modeling effort to be quite successful, as our calculated cross sections do agree favorably with experimentally measured ones in this region of interest. It should be kept in mind that for comparisons to neutron induced experimental cross sections we are considering compound nuclear systems for which the important input parameters to our reaction model (e.g. those that affect level densities and photon-transmission coefficients) are often determined by normalization to experimental data (e.g. from resonance analysis), and so one would expect the comparisons to be good. Since these compund nuclei often bracket the systems of most interest to us, namely those which account for the dominant destruction reactions like $(n, \gamma)$, our systematics should reasonably provide for similar agreement. The proof will have to wait until techniques to measure cross sections on unstable targets are developed in the future. Such efforts are underway at LLNL using a surrogate reaction measurement method (Younes \& Britt 2003) which is the subject of a current LDRD effort in N-Division. In particular, a proof of principle experiment to measure ${ }^{85} \mathrm{Kr}(\mathrm{n}, \gamma){ }^{86} \mathrm{Kr}$ should be carried out by the end of 2005 .

\section{Acknowledgments}

I gratefully acknowledge the helpful suggestions of Ken Moody, Yves Dardenne, Carol Velsko, and Margie Drake in improving this manuscript. This work was performed under the auspices of the U.S. Department of Energy by the University of California Lawrence Livermore National Laboratory under contract W-7405-ENG-48.

\section{REFERENCES}

Avrigeanu, M. \& Avrigeanu, V. IPNE-Bucharest Report No. NP-86-1995 (September 1995) http://www.nea.fr/abs/html/iaea0971.html

Bao, Z. Y., Beer, H., Kappeler, F.,, Voss, F., \& Wisshak, K. 2000, Atomic Data \& Nuclear Data Tables, 76, 70 
Bauer, R. 2002, private communication.

Beer, H., Voss, F., \& Winters, R. R. 1992, ApJS, 80, 403

Bohr, A., \& Mottelson, B. Nuclear Structure Vol. 1, Single-Particle Motion, World Scientific, 1998

Experimental Nuclear Reaction Data File, Brookhaven National Laboratory, US Dept. of Energy, http://www.nndc.bnl.gov/nndc/exfor/

Chadwick, M. 1998, the GNASH statistical model code, http://www.nea.fr/abs/html/psr0125.html

Cline, C. K. 1972, Nucl. Phys. A195, 353

ENSDF: Evaluated Nuclear Structure Data File, Brookhaven National Laboratory, US Dept. of Energy, http://www.nndc.bnl.gov/nndc/ensdf/

Gilbert, A., \& Cameron, A.G.W. 1965, Can. J. Phys., 43, 1446

Demetriou, P., \& Goriely, S. 2001, Nucl. Phys. A695, 95

Hilaire, S., Lagrange, Ch., \& Koning, A. J. 2003, Ann. of Phys. 306, 209

Hofmann, H.M., Richert, J, Tepel, J. W. \& Weidenmuller, H.A. 1975, Ann. of Phys. 90, 403

Hoffman, R. D., Rauscher, T., Woosley, S. E. \& Thielemann, F.-K. 1999, ApJ, 521, 735

Iljinov, A.S., Mebel, M.V., Bianchi, N., De Sanctis, E., Guaraldo, C., Lucherini, V., Muccifora, V., Polli, E., Reolon, A.R., \& Rossi, P. 1992, Nucl. Phys. A543, 517

Keisch, B. 1963, Phys. Rev. 129, 769

Koning, A.J., \& Delaroche, J.P. 2003, Nucl. Phys. A713, 231

Mclane, V., Dunford, C., \& Rose, P. Neutron Cross Sections, Vol. 2, Neutron Cross Section Curves, Academic Press, 1988

Moldauer, P. A. 1976 Phys. Rev. C14 764
Möller, P., Nix, J.R., Myers, W.D., \& Swiatecki, W.J. 1995, Atomic Data \& Nuclear Data Tables, 59, 185

Nethaway, D.R., Memo, "The Cross-Section Sets Used With the Watusi Program." 5 Nov., 1998

Rauscher, T., Thielemann, F.-K., \& Kratz, K.-L. 1997, Phys. Rev. C., 56, 1613

Rauscher, T., Thielemann, F.-K. 2000, Atomic Data \& Nuclear Data Tables, 79, 47

Rauscher, T., Heger, A. Hoffman, R. D. \& Woosley, S. E. 2002, ApJ, 576, 323

Reffo, G. 1978, ICTP Lecture Series, 17 Jan. - 10 Mar., Trieste

Raynal, J. "ECIS96", Proceedings of the Specialists' Meeting on the Nucleon $\mathrm{Nu}$ cleus Optical Model up to $200 \mathrm{MeV}, 13-15$ November 1996, Bruyeres-le-Chatel, Franc (http://www.nea.fr/html/science/om200/raynal.pdf)

Handbook for calculations of nuclear reaction data, Reference input parameter library. 1998, IAEATECDOC-1034

Timmes, F. X., Woosley, S. E., \& Weaver, T. A. 1995, ApJS 98,617

Woosley, S. E., Fowler, W. A., Holmes, J. A. \& Zimmerman, B. A. 1978, Atomic Data \& Nuclear Data Tables, 22, 371

Woosley, S. E., \& Weaver, T. A. 1995, ApJS 101 , 181

Uhl, M., \& Strohmaier, B. IRK-Vienna Report IRK-76/01 1976 (Upd. 1978)

Vonach, H. 1982, UCID-19549, LLNL

Walter, G. et. al. 1986, Nucl Sci. Eng., 93, 357

West, H., Nuckolls, R., Hudson, B., Ruiz, B., Lanier, R., and Mustafa, M. 1993, Phys. Rev. C., 47, 248

Williams, F. C. Jr. 1970, Phys. Lett. 31B, 184

Younes, W. \& Britt, H. C. 2003, Phys. Rev. C67, 024610

This 2-column preprint was prepared with the AAS LATEX macros v5.0. 
A. Basic Nuclear Structure Data

A.1. New Se-Br-Kr Detector Set 


\begin{tabular}{|c|c|c|c|c|c|c|c|c|}
\hline${ }^{A} Z$ & lifetime & $(\mathrm{n}, 2 \mathrm{n})$ & $\left(\mathrm{n}, \mathrm{n}^{\prime}\right)$ & $(\mathrm{n}, \gamma)$ & $(\mathrm{n}, \mathrm{p})$ & $(n, n p)$ & $(\mathrm{p}, \mathrm{n})$ & $(\mathrm{p}, 2 \mathrm{n})$ \\
\hline${ }^{74} \mathrm{Se}$ & $0.89 \%$ & & & $\bullet$ & & & $\bullet$ & \\
\hline${ }^{75} \mathrm{Se}$ & $120 \mathrm{~d}$ & - & & - & & & - & - \\
\hline${ }^{76} \mathrm{Se}$ & $9.4 \%$ & - & & - & & & - & - \\
\hline${ }^{77} \mathrm{Se}$ & $7.6 \%$ & - & & - & & & - & - \\
\hline${ }^{77} \mathrm{Se}_{m}$ & $17.4 \mathrm{~s}$ & - & - & - & & & - & - \\
\hline${ }^{78} \mathrm{Se}$ & $23.8 \%$ & - & & - & & & - & - \\
\hline${ }^{79} \mathrm{Se}$ & $6.5 \times 10^{4} \mathrm{y}$ & • & & $\bullet$ & & & - & - \\
\hline${ }^{79} \mathrm{Se}_{m}$ & $3.91 \mathrm{~m}$ & - & - & - & & & - & - \\
\hline${ }^{80} \mathrm{Se}$ & $49.6 \%$ & - & & - & & & - & - \\
\hline${ }^{81} \mathrm{Se}$ & $18.5 \mathrm{~m}$ & - & & - & & & - & - \\
\hline${ }^{81} \mathrm{Se}_{m}$ & $57.3 \mathrm{~m}$ & - & - & - & & & - & - \\
\hline${ }^{75} \mathrm{Br}$ & $97.6 \mathrm{~m}$ & & & $\bullet$ & $\bullet$ & $\bullet$ & & \\
\hline${ }^{76} \mathrm{Br}$ & $16.2 \mathrm{~h}$ & $\bullet$ & & - & - & - & - & \\
\hline${ }^{76} \mathrm{Br}_{m}$ & $1.31 \mathrm{~s}$ & - & - & - & - & - & - & \\
\hline${ }^{77} \mathrm{Br}$ & $57.0 \mathrm{~h}$ & - & & - & - & - & - & - \\
\hline${ }^{77} \mathrm{Br}_{m}$ & $4.28 \mathrm{~m}$ & - & - & - & - & - & - & - \\
\hline${ }^{78} \mathrm{Br}$ & $6.46 \mathrm{~m}$ & - & & - & - & - & - & - \\
\hline${ }^{78} \mathrm{Br}_{m}$ & $1.2 \times 10^{-4} \mathrm{~s}$ & - & - & - & - & - & - & - \\
\hline${ }^{79} \mathrm{Br}$ & $50.69 \%$ & - & & - & - & - & - & - \\
\hline${ }^{79} \mathrm{Br}_{m}$ & $4.86 \mathrm{~s}$ & - & - & - & - & - & - & - \\
\hline${ }^{80} \mathrm{Br}$ & $17.68 \mathrm{~m}$ & - & & - & - & - & - & - \\
\hline${ }^{80} \mathrm{Br}_{m}$ & $4.42 \mathrm{~h}$ & - & - & - & - & - & - & - \\
\hline${ }^{81} \mathrm{Br}$ & $49.31 \%$ & - & & - & - & - & - & - \\
\hline${ }^{81} \mathrm{Br}_{m}$ & $3.5 \times 10^{-5} \mathrm{~s}$ & $\bullet$ & - & - & - & - & - & - \\
\hline${ }^{82} \mathrm{Br}$ & $35.3 \mathrm{~h}$ & - & & - & & - & - & - \\
\hline${ }^{82} \mathrm{Br}_{m}$ & $6.13 \mathrm{~m}$ & - & - & - & & - & - & - \\
\hline${ }^{76} \mathrm{Kr}$ & $14.8 \mathrm{~h}$ & & & $\bullet$ & $\bullet$ & $\bullet$ & & \\
\hline${ }^{77} \mathrm{Kr}$ & $74.4 \mathrm{~m}$ & - & & - & - & - & - & \\
\hline${ }^{78} \mathrm{Kr}$ & $0.35 \%$ & - & & - & - & - & - & - \\
\hline${ }^{79} \mathrm{Kr}$ & $35.04 \mathrm{~h}$ & - & & - & - & - & - & - \\
\hline${ }^{79} \mathrm{Kr}_{m}$ & $50.0 \mathrm{~s}$ & - & - & - & - & - & - & - \\
\hline${ }^{80} \mathrm{Kr}$ & $2.25 \%$ & - & & - & - & - & - & - \\
\hline${ }^{81} \mathrm{Kr}$ & $2.1 \times 10^{5} \mathrm{y}$ & - & & - & - & - & - & - \\
\hline${ }^{81} \mathrm{Kr}_{m}$ & $13.0 \mathrm{~s}$ & - & - & - & - & - & - & - \\
\hline${ }^{82} \mathrm{Kr}$ & $11.6 \%$ & - & & - & - & - & - & - \\
\hline${ }^{83} \mathrm{Kr}$ & $11.5 \%$ & - & & - & & - & - & - \\
\hline${ }^{83} \mathrm{Kr}_{m}$ & $1.83 \mathrm{~h}$ & - & - & - & & - & - & - \\
\hline${ }^{77} \mathrm{Rb}$ & $3.8 \mathrm{~m}$ & & & $\bullet$ & $\bullet$ & $\bullet$ & & \\
\hline${ }^{78} \mathrm{Rb}$ & $17.7 \mathrm{~m}$ & - & & - & - & - & & \\
\hline${ }^{78} \mathrm{Rb}_{m}$ & $5.74 \mathrm{~m}$ & - & - & - & - & - & & \\
\hline${ }^{79} \mathrm{Rb}$ & $22.9 \mathrm{~m}$ & - & & - & - & - & & \\
\hline${ }^{80} \mathrm{Rb}$ & $34.0 \mathrm{~s}$ & - & & - & - & - & & \\
\hline${ }^{81} \mathrm{Rb}$ & $4.6 \mathrm{~h}$ & - & & - & - & - & & \\
\hline${ }^{81} \mathrm{Rb}_{m}$ & $30.5 \mathrm{~m}$ & 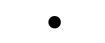 & - & - & - & - & & \\
\hline${ }^{82} \mathrm{Rb}$ & $1.27 \mathrm{~m}$ & - & & - & - & - & & \\
\hline${ }^{82} \mathrm{Rb}_{m}$ & $6.47 \mathrm{~h}$ & $\bullet$ & $\bullet$ & $\bullet$ & $\bullet$ & - & & \\
\hline${ }^{83} \mathrm{Rb}$ & $86.2 \mathrm{~d}$ & - & & - & - & - & & \\
\hline${ }^{84} \mathrm{Rb}$ & $32.8 \mathrm{~d}$ & 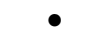 & & - & & - & & \\
\hline${ }^{84} \mathrm{Rb}_{m}$ & $20.3 \mathrm{~m}$ & $\bullet$ & - & - & & - & & \\
\hline
\end{tabular}

Table 2: Reactions calculated for new Br-Kr set. 
A.2. Binding and Separation Energies 


\begin{tabular}{|c|c|c|c|c|c|c|}
\hline${ }^{A} Z$ & $J^{\pi}$ & $\mathrm{BE}(\mathrm{MeV})$ & $S_{n}(\mathrm{MeV})$ & $S_{p}(\mathrm{MeV})$ & $S_{\alpha}(\mathrm{MeV})$ & $S_{d}(\mathrm{MeV})$ \\
\hline${ }^{74} \mathrm{Se}$ & $0+$ & 642.88 & 8.03 & 4.22 & 4.37 & 11.22 \\
\hline${ }^{75} \mathrm{Se}$ & $5 / 2+$ & 650.91 & 11.15 & 5.41 & 4.69 & 14.20 \\
\hline${ }^{76} \mathrm{Se}$ & $0+$ & 662.06 & 7.42 & 5.28 & 5.06 & 11.34 \\
\hline${ }^{77} \mathrm{Se}$ & $1 / 2-$ & 669.48 & 10.50 & 6.14 & 5.51 & 14.61 \\
\hline${ }^{77} \mathrm{Se}_{m}$ & $7 / 2+$ & & & & & \\
\hline${ }^{78} \mathrm{Se}$ & $0+$ & 679.98 & 6.96 & 6.33 & 5.98 & 12.00 \\
\hline${ }^{79} \mathrm{Se}$ & $7 / 2+$ & 686.94 & 9.91 & 7.26 & 6.48 & 15.19 \\
\hline${ }^{79} \mathrm{Se}_{m}$ & $1 / 2$ & & & & & \\
\hline${ }^{80} \mathrm{Se}$ & $0+$ & 696.85 & 6.70 & 7.50 & 7.09 & 12.88 \\
\hline${ }^{81} \mathrm{Se}$ & $1 / 2-$ & 703.55 & 9.28 & 8.40 & 7.51 & 15.76 \\
\hline${ }^{81} \mathrm{Se}_{m}$ & $7 / 2+$ & & & & & \\
\hline${ }^{82} \mathrm{Se}$ & $0+$ & 712.83 & 5.81 & 8.70 & 8.08 & 13.32 \\
\hline${ }^{75} \mathrm{Br}$ & $3 / 2-$ & 647.09 & 9.22 & 7.13 & 4.07 & 14.17 \\
\hline${ }^{76} \mathrm{Br}$ & $1-$ & 656.32 & 11.02 & 7.17 & 4.29 & 17.01 \\
\hline${ }^{76} \mathrm{Br}_{m}$ & $(4)+$ & & & & & \\
\hline${ }^{77} \mathrm{Br}$ & $3 / 2$ & 667.34 & 8.28 & 8.21 & 4.64 & 14.34 \\
\hline${ }^{77} \mathrm{Br}_{m}$ & $9 / 2+$ & & & & & \\
\hline${ }^{78} \mathrm{Br}$ & $1+$ & 675.62 & 10.69 & 8.28 & 5.18 & 17.58 \\
\hline${ }^{78} \mathrm{Br}_{m}$ & $4+$ & & & & & \\
\hline${ }^{79} \mathrm{Br}$ & $3 / 2-$ & 686.31 & 7.89 & 9.11 & 5.43 & 14.76 \\
\hline${ }^{79} \mathrm{Br}_{m}$ & $9 / 2+$ & & & & & \\
\hline${ }^{80} \mathrm{Br}$ & $1+$ & 694.20 & 10.15 & 9.09 & 6.28 & 17.83 \\
\hline${ }^{80} \mathrm{Br}_{m}$ & 5- & & & & & \\
\hline${ }^{81} \mathrm{Br}$ & $3 / 2-$ & 704.35 & 7.60 & 9.91 & 6.62 & 15.15 \\
\hline${ }^{81} \mathrm{Br}_{m}$ & $9 / 2+$ & & & & & \\
\hline${ }^{82} \mathrm{Br}$ & $5-$ & 711.95 & 9.58 & 9.77 & 7.67 & 18.07 \\
\hline${ }^{82} \mathrm{Br}_{m}$ & $2-$ & & & & & \\
\hline${ }^{83} \mathrm{Br}$ & $(3 / 2)-$ & 721.53 & 6.84 & 10.71 & 8.00 & 15.60 \\
\hline${ }^{76} \mathrm{Kr}$ & $0+$ & 654.22 & 9.26 & 3.23 & 3.64 & 11.10 \\
\hline${ }^{77} \mathrm{Kr}$ & $5 / 2+$ & 663.48 & 12.06 & 4.06 & 3.72 & 13.76 \\
\hline${ }^{78} \mathrm{Kr}$ & $0+$ & 675.55 & 8.35 & 3.92 & 4.26 & 11.14 \\
\hline${ }^{79} \mathrm{Kr}$ & $1 / 2-$ & 683.90 & 11.52 & 5.01 & 4.78 & 14.16 \\
\hline${ }^{79} \mathrm{Kr}_{m}$ & $7 / 2+$ & & & & & \\
\hline${ }^{80} \mathrm{Kr}$ & $0+$ & 695.42 & 7.87 & 4.86 & 5.17 & 11.45 \\
\hline${ }^{81} \mathrm{Kr}$ & $7 / 2+$ & 703.29 & 10.97 & 5.81 & 5.83 & 14.53 \\
\hline${ }^{81} \mathrm{Kr}_{m}$ & $1 / 2-$ & & & & & \\
\hline${ }^{82} \mathrm{Kr}$ & $0+$ & 714.26 & 7.46 & 5.78 & 6.35 & 12.30 \\
\hline${ }^{83} \mathrm{Kr}$ & $9 / 2+$ & 721.72 & 10.52 & 7.06 & 7.32 & 15.32 \\
\hline${ }^{83} \mathrm{Kr}_{m}$ & $1 / 2-$ & & & & & \\
\hline${ }^{84} \mathrm{Kr}$ & $0+$ & 732.24 & 7.12 & 7.03 & 7.91 & 13.45 \\
\hline${ }^{77} \mathrm{Rb}$ & $3 / 2-$ & 657.45 & 10.09 & 6.43 & 3.45 & 14.37 \\
\hline${ }^{78} \mathrm{Rb}$ & $0(+)$ & 667.54 & 11.92 & 6.50 & 3.66 & 16.40 \\
\hline${ }^{78} \mathrm{Rb}_{m}$ & $4(-)$ & & & & & \\
\hline${ }^{79} \mathrm{Rb}$ & $5 / 2+$ & 679.47 & 9.44 & 6.70 & 3.97 & 13.81 \\
\hline${ }^{80} \mathrm{Rb}$ & $1+$ & 688.91 & 11.37 & 6.60 & 4.49 & 16.98 \\
\hline${ }^{81} \mathrm{Rb}$ & $3 / 2-$ & 700.28 & 8.82 & 7.83 & 4.81 & 14.48 \\
\hline${ }^{81} \mathrm{Rb}_{m}$ & $9 / 2+$ & & & & & \\
\hline${ }^{82} \mathrm{Rb}$ & $1+$ & 709.10 & 10.94 & 7.88 & 5.49 & 17.57 \\
\hline${ }^{82} \mathrm{Rb}_{m}$ & 5- & & & & & \\
\hline${ }^{83} \mathrm{Rb}$ & $5 / 2-$ & 720.04 & 8.74 & 8.85 & 6.35 & 15.16 \\
\hline${ }^{84} \mathrm{Rb}$ & $2-$ & 728.78 & 10.49 & 8.64 & 6.97 & 17.91 \\
\hline${ }^{84} \mathrm{Rb}_{m}$ & $6-$ & & & & & \\
\hline${ }^{85} \mathrm{Rb}$ & $5 / 2-$ & 739.27 & 8.65 & 9.64 & 7.95 & 15.85 \\
\hline
\end{tabular}

Table 3: $J^{\pi}$, Binding, and Separation Energies for new Br-Kr set. 


\section{A.3. Q-values for Select Reactions}

\begin{tabular}{|c|c|c|c|c|c|c|c|}
\hline${ }^{A} Z$ & $(\mathrm{n}, 2 \mathrm{n})$ & $(\mathrm{n}, \mathrm{p})$ & $(\mathrm{n}, \mathrm{np})$ & $(\mathrm{p}, \mathrm{n})$ & $(\mathrm{p}, 2 \mathrm{n})$ & $(\mathrm{d}, \mathrm{n})$ & $(\mathrm{d}, 2 \mathrm{n})$ \\
\hline${ }^{74} \mathrm{Se}$ & -12.06 & -0.57 & -8.54 & -7.69 & -17.50 & 1.99 & -9.92 \\
\hline${ }^{75} \mathrm{Se}$ & -8.03 & 1.64 & -8.60 & -3.81 & -15.72 & 3.18 & -6.04 \\
\hline${ }^{76} \mathrm{Se}$ & -11.15 & -2.18 & -9.51 & -5.74 & -14.96 & 3.05 & -7.97 \\
\hline${ }^{77} \mathrm{Se}$ & -7.42 & 0.10 & -9.60 & -2.14 & -13.16 & 3.91 & -4.37 \\
\hline${ }^{78} \mathrm{Se}$ & -10.50 & -3.43 & -10.40 & -4.36 & -12.64 & 4.10 & -6.59 \\
\hline${ }^{79} \mathrm{Se}$ & -6.96 & -1.50 & -10.39 & -0.63 & -11.32 & 5.03 & -2.86 \\
\hline${ }^{80} \mathrm{Se}$ & -9.91 & -4.86 & -11.41 & -2.65 & -10.54 & 5.27 & -4.88 \\
\hline${ }^{81} \mathrm{Se}$ & -6.70 & -3.08 & -11.56 & 0.80 & -9.35 & 6.17 & -1.43 \\
\hline${ }^{82} \mathrm{Se}$ & -9.28 & -6.58 & -12.36 & -0.88 & -8.48 & 6.47 & -3.11 \\
\hline${ }^{75} \mathrm{Br}$ & -11.91 & 3.81 & -4.22 & -5.68 & -15.82 & 4.90 & -7.91 \\
\hline${ }^{76} \mathrm{Br}$ & -9.22 & 5.74 & -5.41 & -2.09 & -14.90 & 4.94 & -4.32 \\
\hline${ }^{77} \mathrm{Br}$ & -11.02 & 2.14 & -5.28 & -3.85 & -13.11 & 5.98 & -6.08 \\
\hline${ }^{78} \mathrm{Br}$ & -8.28 & 4.36 & -6.14 & -0.07 & -12.13 & 6.05 & -2.30 \\
\hline${ }^{79} \mathrm{Br}$ & -10.69 & 0.63 & -6.33 & -2.41 & -10.76 & 6.88 & -4.64 \\
\hline${ }^{80} \mathrm{Br}$ & -7.89 & 2.65 & -7.26 & 1.22 & -10.30 & 6.86 & -1.01 \\
\hline${ }^{81} \mathrm{Br}$ & -10.15 & -0.80 & -7.50 & -1.06 & -8.93 & 7.68 & -3.29 \\
\hline${ }^{82} \mathrm{Br}$ & -7.60 & 0.88 & -8.40 & 2.31 & -8.66 & 7.54 & 0.08 \\
\hline${ }^{83} \mathrm{Br}$ & -9.58 & -2.89 & -8.70 & 0.19 & -7.27 & 8.48 & -2.04 \\
\hline${ }^{76} \mathrm{Kr}$ & -12.81 & 2.09 & $\begin{array}{l}-7.13 \\
\end{array}$ & $\begin{array}{l}-9.19 \\
\end{array}$ & -20.55 & 1.00 & -11.42 \\
\hline${ }^{77} \mathrm{Kr}$ & -9.26 & 3.85 & -7.17 & -6.03 & -18.45 & 1.83 & -8.26 \\
\hline${ }^{78} \mathrm{Kr}$ & -12.06 & 0.07 & -8.21 & -8.00 & -18.09 & 1.69 & -10.23 \\
\hline${ }^{79} \mathrm{Kr}$ & -8.35 & 2.41 & -8.28 & -4.43 & -16.35 & 2.78 & -6.66 \\
\hline${ }^{80} \mathrm{Kr}$ & -11.52 & -1.22 & -9.11 & -6.51 & -15.95 & 2.63 & -8.74 \\
\hline${ }^{81} \mathrm{Kr}$ & -7.87 & 1.06 & -9.09 & -3.01 & -14.38 & 3.58 & -5.24 \\
\hline${ }^{82} \mathrm{Kr}$ & -10.97 & -2.31 & -9.91 & -5.16 & -13.98 & 3.55 & -7.39 \\
\hline${ }^{83} \mathrm{Kr}$ & -7.46 & -0.19 & -9.77 & -1.68 & -12.62 & 4.83 & -3.91 \\
\hline${ }^{84} \mathrm{Kr}$ & -10.52 & -3.87 & -10.71 & -3.46 & -12.20 & 4.80 & -5.69 \\
\hline${ }^{77} \mathrm{Rb}$ & $\begin{array}{c}-12.42 \\
\end{array}$ & 6.03 & \begin{tabular}{|l|}
-3.23 \\
\end{tabular} & $\begin{array}{l}-7.81 \\
\end{array}$ & -18.80 & 4.20 & -10.04 \\
\hline${ }^{78} \mathrm{Rb}$ & -10.09 & 8.00 & -4.06 & -3.66 & -17.90 & 4.27 & -5.89 \\
\hline${ }^{79} \mathrm{Rb}$ & -11.92 & 4.43 & -3.92 & -5.42 & -15.58 & 4.47 & -7.65 \\
\hline${ }^{80} \mathrm{Rb}$ & -9.44 & 6.51 & -5.01 & -2.74 & -14.86 & 4.37 & -4.97 \\
\hline${ }^{81} \mathrm{Rb}$ & -11.37 & 3.01 & -4.86 & -4.77 & -14.11 & 5.60 & -7.00 \\
\hline${ }^{82} \mathrm{Rb}$ & -8.82 & 5.16 & -5.81 & -0.99 & -13.59 & 5.65 & -3.22 \\
\hline${ }^{83} \mathrm{Rb}$ & -10.94 & 1.68 & -5.78 & -3.06 & -11.93 & 6.62 & -5.29 \\
\hline${ }^{84} \mathrm{Rb}$ & -8.74 & 3.46 & -7.06 & 0.11 & -11.80 & 6.41 & -2.12 \\
\hline${ }^{85} \mathrm{Rb}$ & -10.49 & 0.09 & -7.03 & -1.85 & -10.38 & 7.41 & -4.08 \\
\hline
\end{tabular}

Table 4: Q-values $(\mathrm{MeV})$ for select reactions in the new Br-Kr set. 
A.4. Adopted Level Schemes 

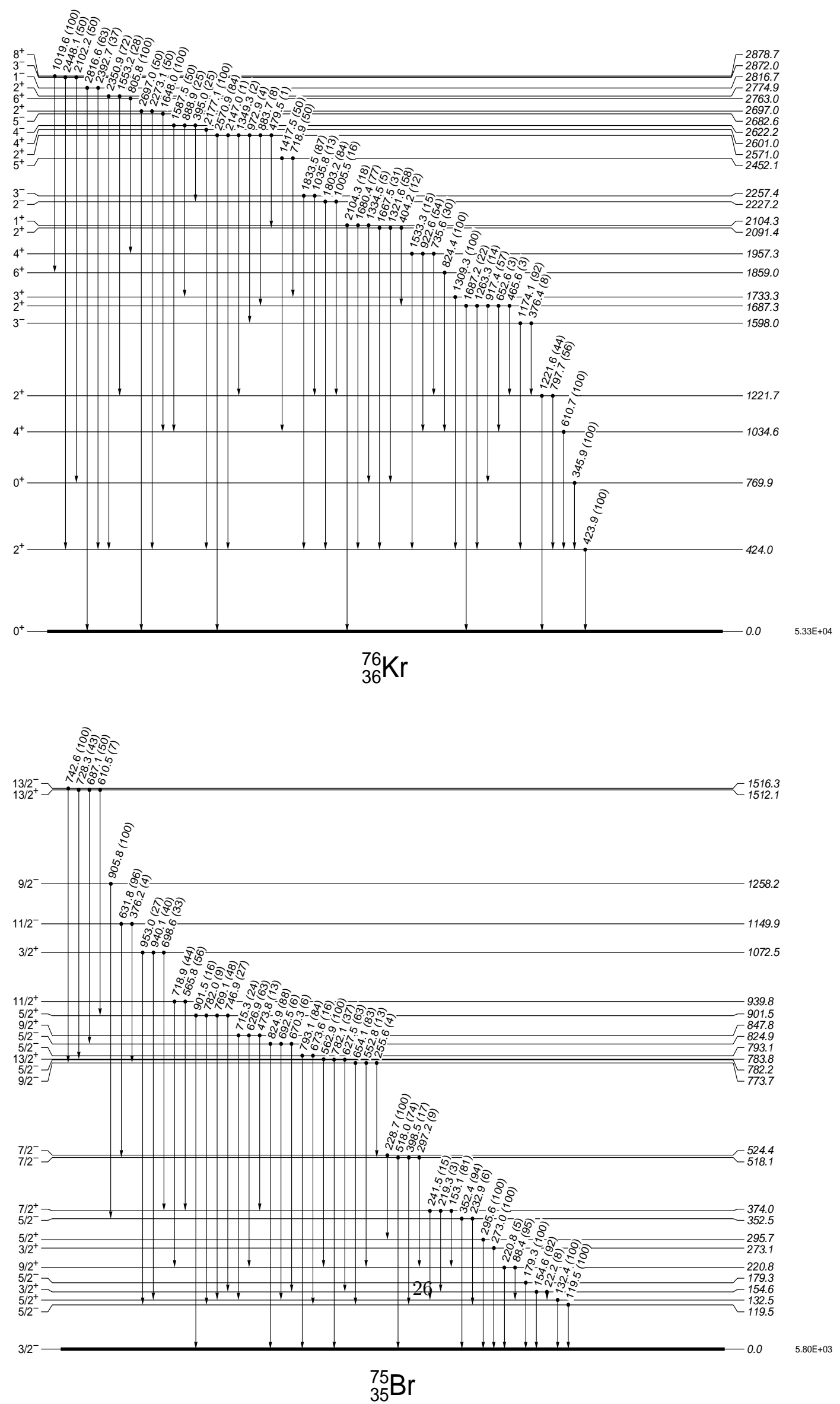

Fig. 11. - Adopted level schemes for $\mathrm{Br}$ and $\mathrm{Kr}$ nuclei, $\mathrm{N}=40$ 

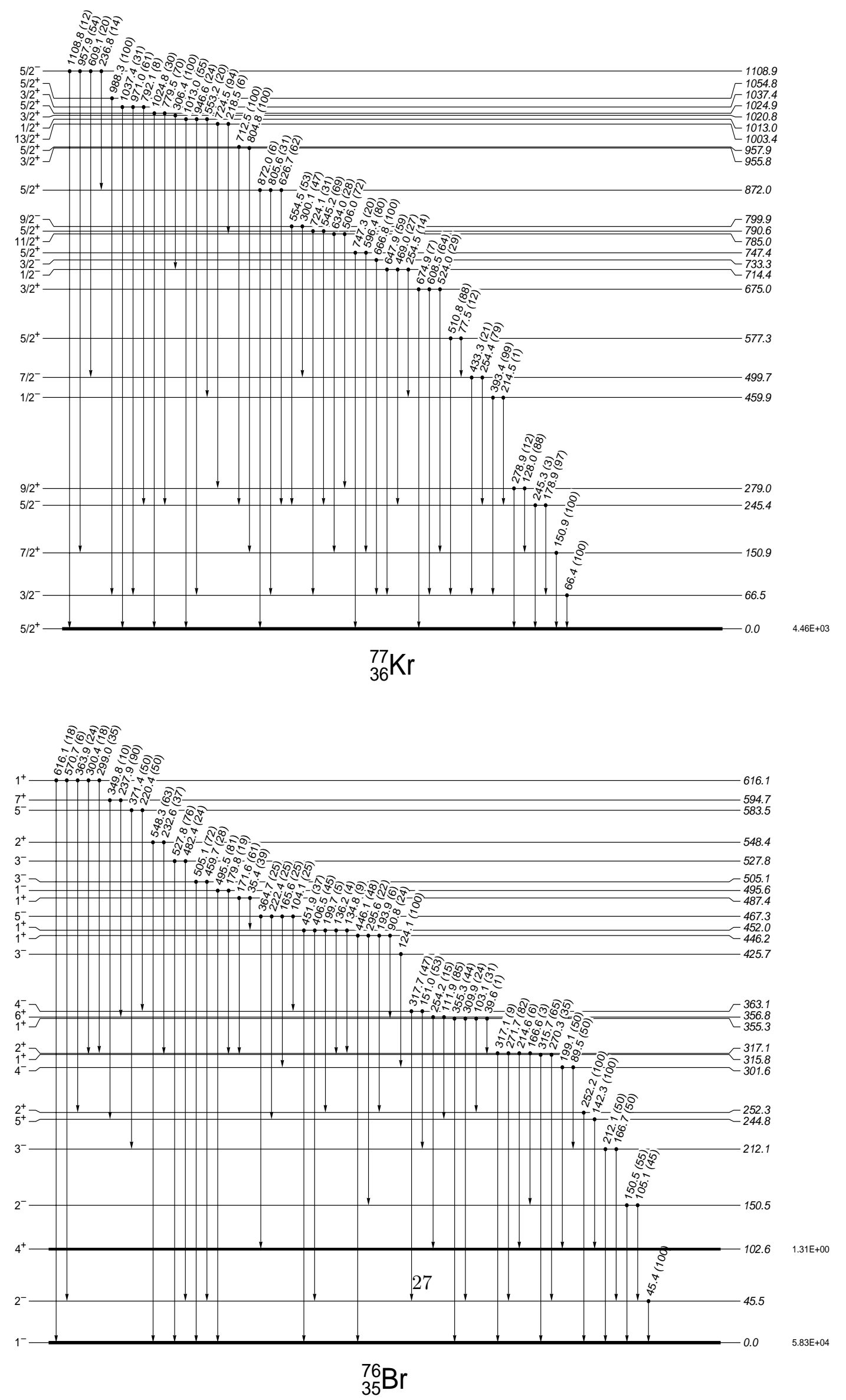

Fig. 12.- Adopted level schemes for $\mathrm{Br}$ and $\mathrm{Kr}$ nuclei, $\mathrm{N}=41$ 

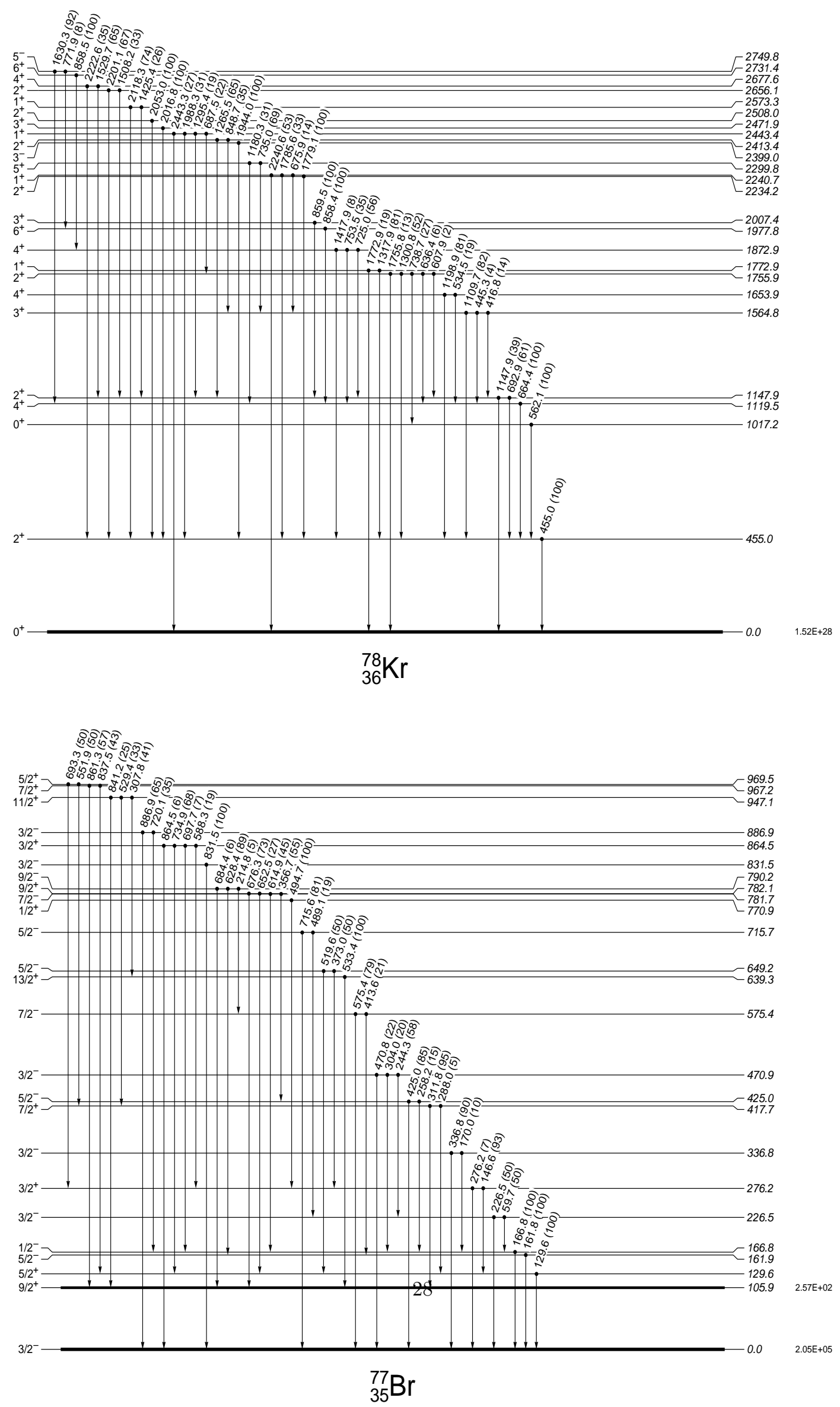

Fig. 13.- Adopted level schemes for $\mathrm{Br}$ and $\mathrm{Kr}$ nuclei, $\mathrm{N}=42$ 

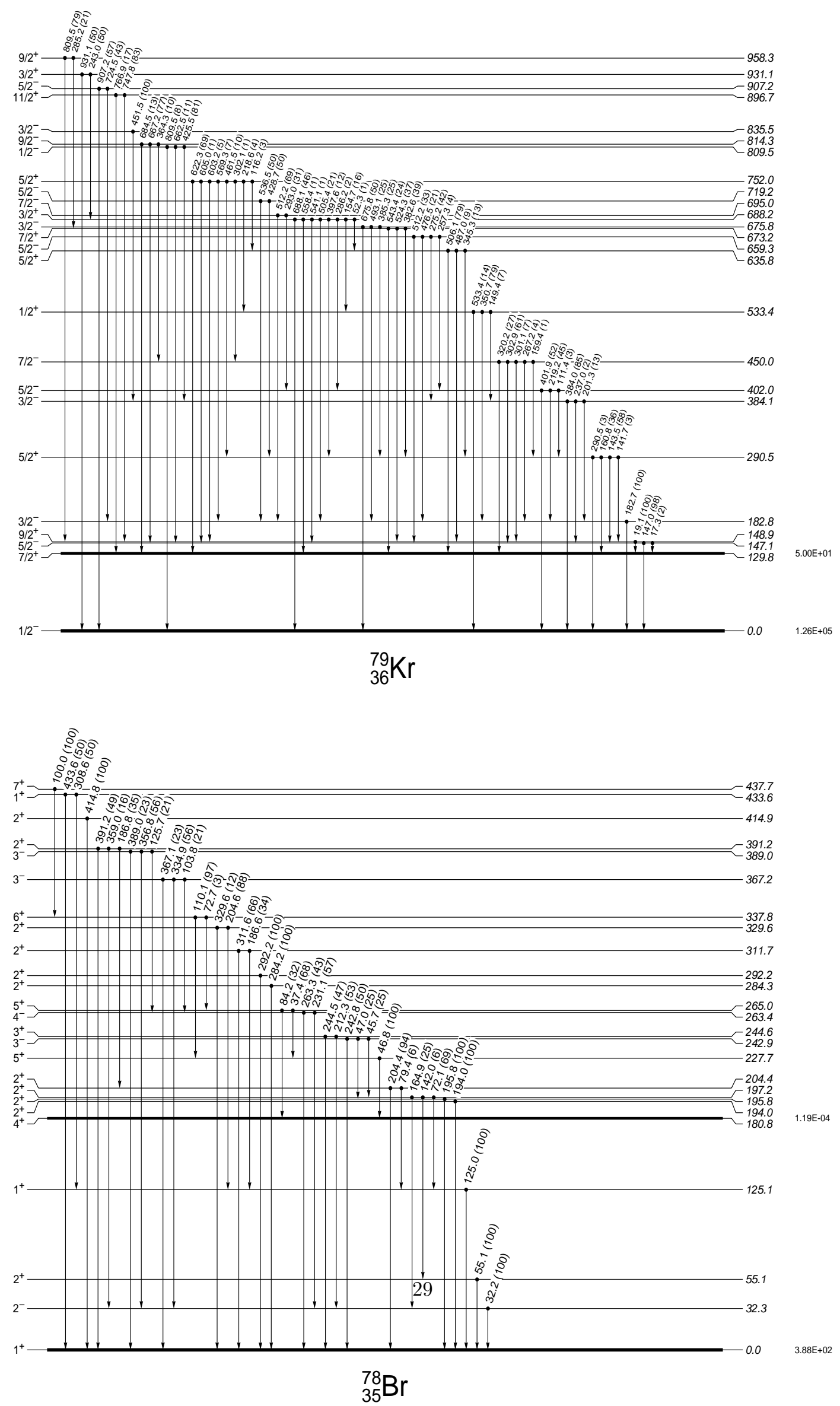

Fig. 14.- Adopted level schemes for $\mathrm{Br}$ and $\mathrm{Kr}$ nuclei, $\mathrm{N}=43$ 

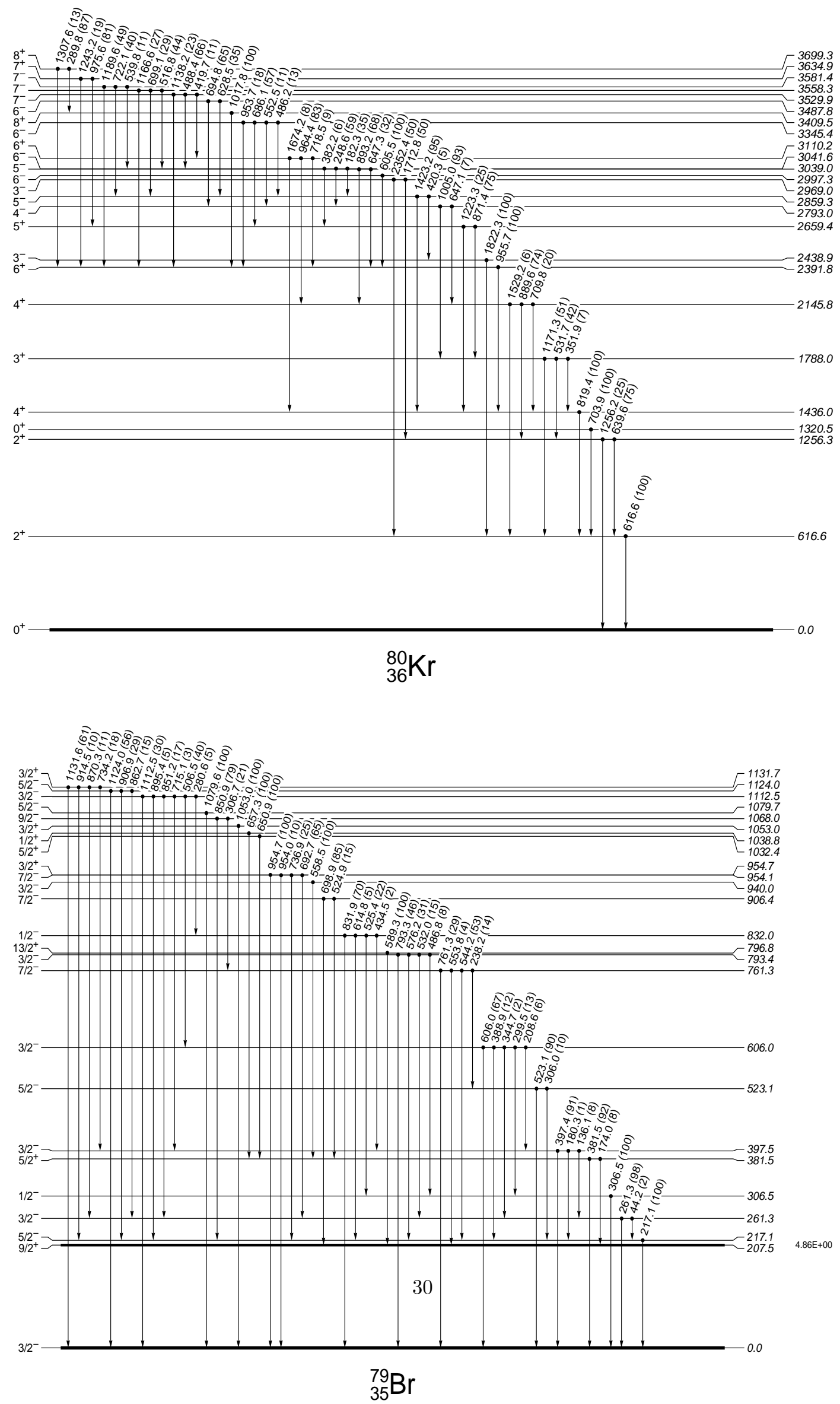

Fig. 15.- Adopted level schemes for $\mathrm{Br}$ and $\mathrm{Kr}$ nuclei, $\mathrm{N}=44$ 

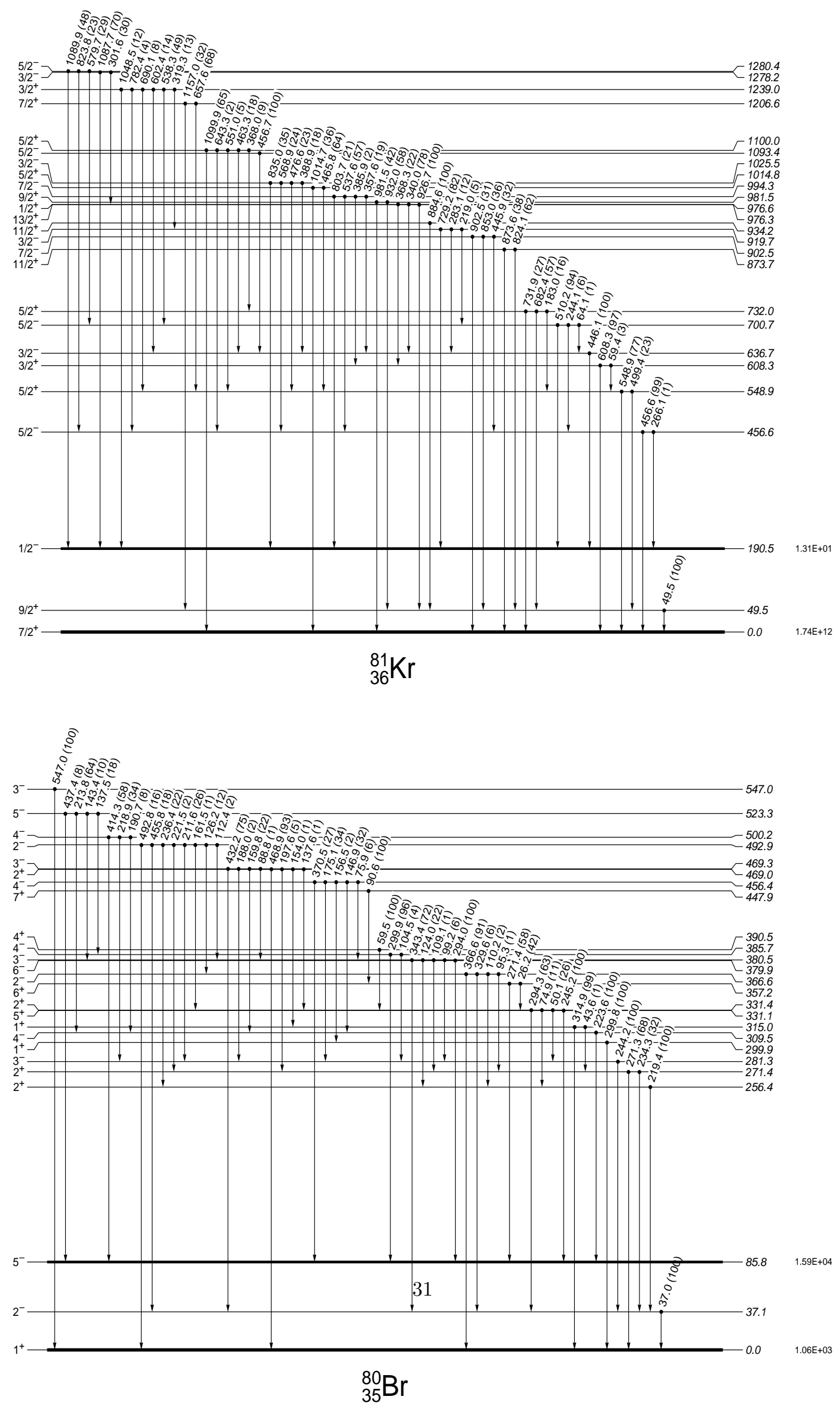

Fig. 16.- Adopted level schemes for $\mathrm{Br}$ and $\mathrm{Kr}$ nuclei, $\mathrm{N}=45$ 

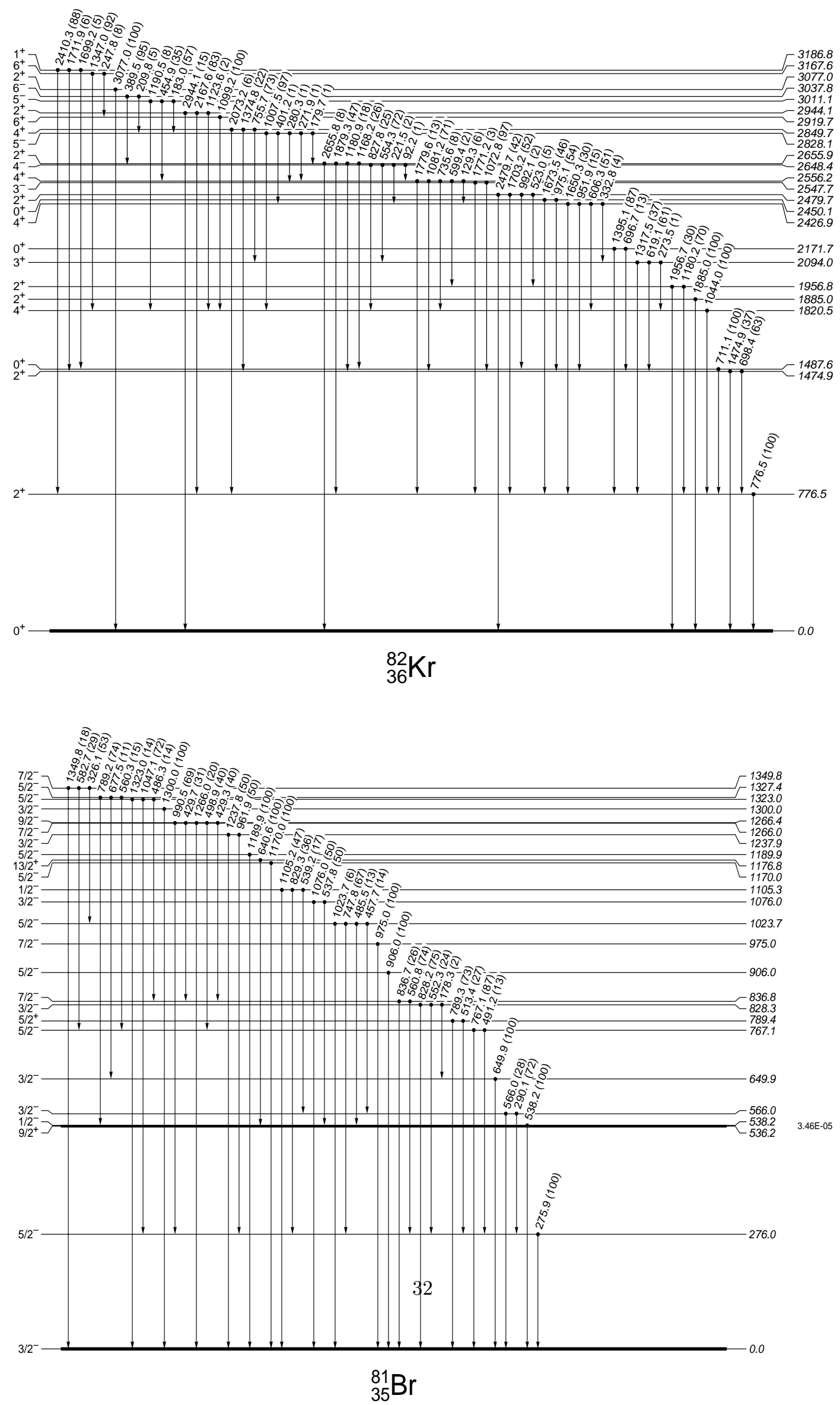

Fig. 17.- Adopted level schemes for $\mathrm{Br}$ and $\mathrm{Kr}$ nuclei, $\mathrm{N}=46$ 

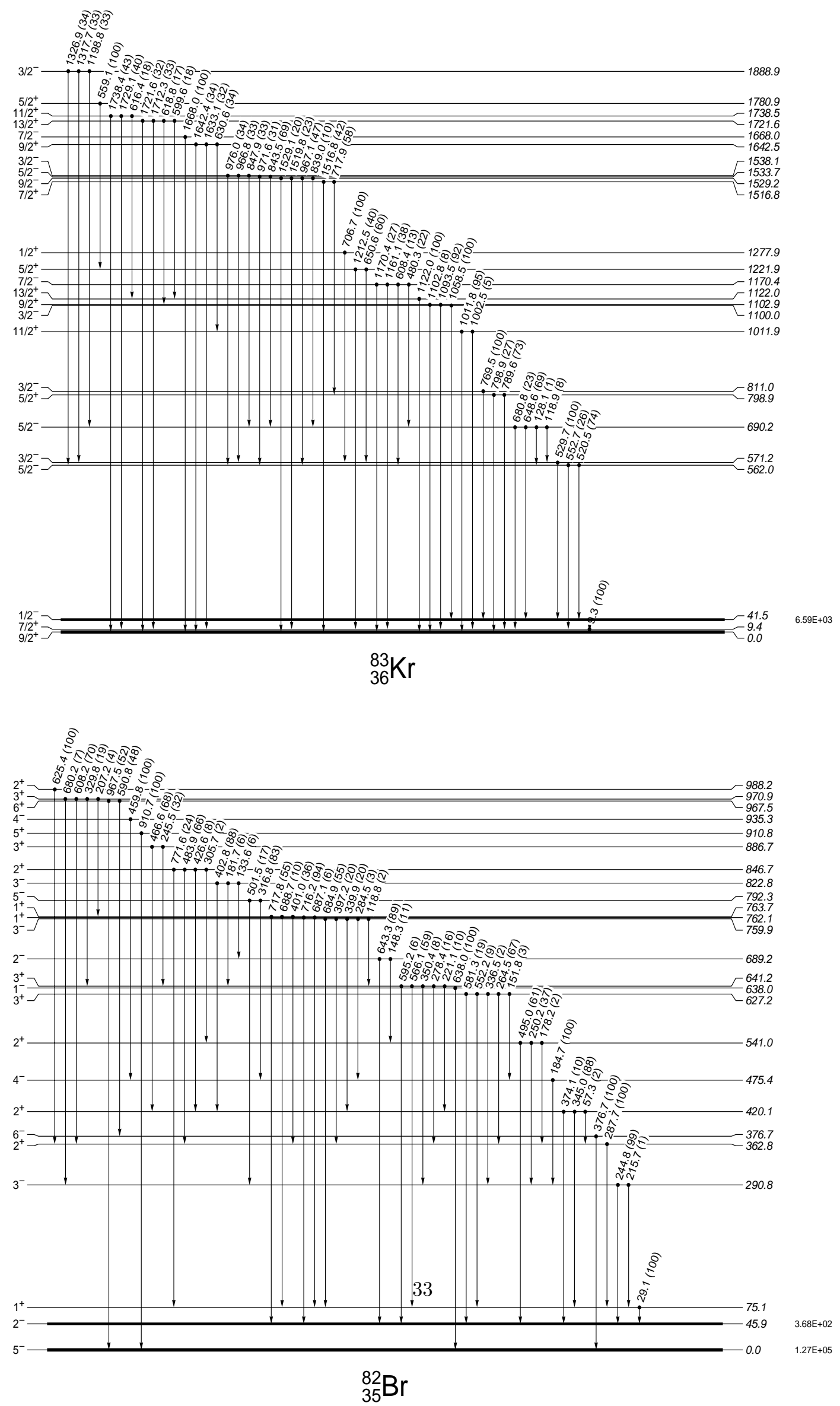

Fig. 18. - Adopted level schemes for $\mathrm{Br}$ and $\mathrm{Kr}$ nuclei, $\mathrm{N}=47$ 

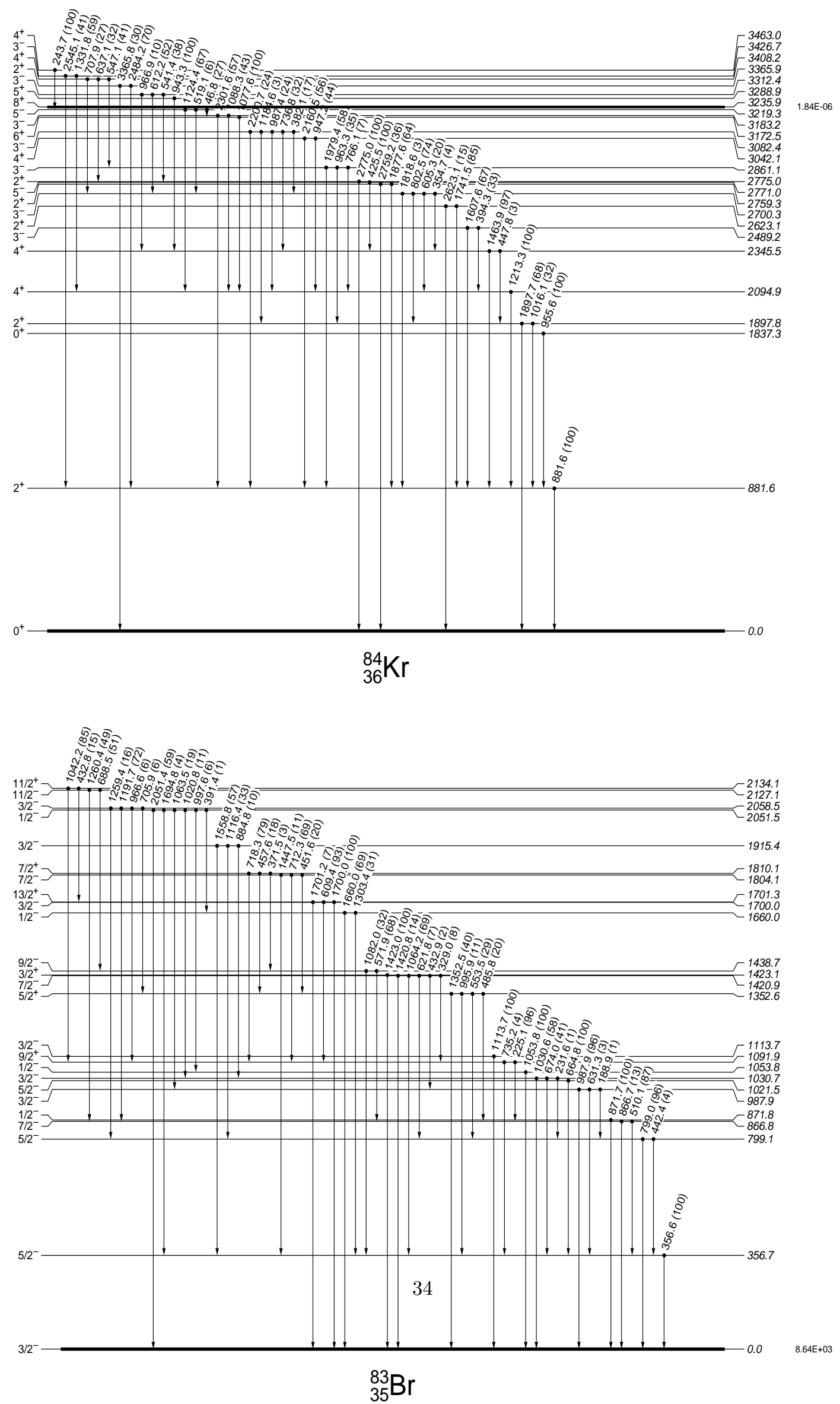

Fig. 19.- Adopted level schemes for $\mathrm{Br}$ and $\mathrm{Kr}$ nuclei, $\mathrm{N}=48$ 
A.5. Nuclear Level Density Parameters 


\begin{tabular}{|c|c|c|c|c|c|c|c|c|c|c|c|c|}
\hline${ }^{A} Z$ & $\begin{array}{r}\tilde{a}(A) \\
(\mathrm{MeV})^{-1}\end{array}$ & $\begin{array}{c}\Delta \\
(\mathrm{MeV})\end{array}$ & $\begin{array}{c}\delta W \\
(\mathrm{MeV})\end{array}$ & $\mathrm{x} / \mathrm{s}$ & $\sigma^{2}\left(E_{x}\right)$ & $\begin{array}{c}E_{x} \\
(\mathrm{MeV})\end{array}$ & $\begin{array}{c}E_{0} \\
(\mathrm{MeV})\end{array}$ & $\begin{array}{c}T \\
(\mathrm{MeV})\end{array}$ & $\mathrm{I} / \mathrm{G}$ & $\mathrm{N}$ & $\begin{array}{c}E_{\text {cut }} \\
(\mathrm{MeV})\end{array}$ & $\sigma^{2}\left(E_{c u t}\right)$ \\
\hline${ }^{73} \mathrm{As}$ & 9.125 & 1.320 & 12.500 & $\mathrm{~s}$ & 17.372 & 5.875 & -2.375 & 0.733 & I & 9 & 0.577 & 3.047 \\
\hline${ }^{74} \mathrm{As}$ & 9.250 & 0.000 & 10.157 & $\mathrm{~s}$ & 16.999 & 4.527 & -3.607 & 0.752 & I & 9 & 0.278 & 4.750 \\
\hline${ }^{75} \mathrm{As}$ & 9.375 & 1.355 & 9.634 & $\mathrm{~s}$ & 18.054 & 6.400 & -2.704 & 0.788 & I & 13 & 0.865 & 3.125 \\
\hline${ }^{76} \mathrm{As}$ & 9.500 & 0.000 & 8.776 & $\mathrm{x}$ & 17.073 & 4.474 & -3.522 & 0.752 & I & 12 & 0.293 & 1.943 \\
\hline${ }^{77} \mathrm{As}$ & 9.625 & 1.490 & 8.588 & $\mathrm{~s}$ & 18.259 & 6.500 & -2.502 & 0.786 & I & 8 & 0.632 & 3.946 \\
\hline${ }^{78} \mathrm{As}$ & 9.750 & 0.000 & 8.065 & $\mathrm{~s}$ & 8.317 & 1.000 & -0.870 & 0.465 & I & 4 & 0.294 & 0.792 \\
\hline${ }^{79} \mathrm{As}$ & 9.875 & 1.330 & 7.542 & $\mathrm{~S}$ & 17.364 & 5.729 & -2.093 & 0.744 & I & 5 & 0.499 & 1.875 \\
\hline${ }^{80} \mathrm{As}$ & 10.000 & 0.000 & 7.019 & $\mathrm{~s}$ & 8.410 & 1.000 & -0.872 & 0.469 & I & 3 & 0.265 & 0.625 \\
\hline${ }^{81} \mathrm{As}$ & 10.125 & 1.125 & 6.496 & $\mathrm{~s}$ & 16.508 & 5.000 & -1.829 & 0.709 & I & 5 & 0.738 & 2.781 \\
\hline${ }^{74} \mathrm{Se}$ & 9.250 & 3.795 & 14.500 & $\mathrm{~S}$ & 20.026 & 9.400 & -0.971 & 0.778 & I & 9 & 2.108 & 4.500 \\
\hline${ }^{75} \mathrm{Se}$ & 9.375 & 1.570 & 12.093 & $\mathrm{x}$ & 19.395 & 7.000 & -2.940 & 0.786 & I & 11 & 0.748 & 4.700 \\
\hline${ }^{76} \mathrm{Se}$ & 9.500 & 3.785 & 11.634 & $\mathrm{~s}$ & 20.867 & 10.000 & -1.470 & 0.836 & I & 7 & 1.788 & 4.292 \\
\hline${ }^{77} \mathrm{Se}$ & 9.625 & 1.615 & 11.672 & $\mathrm{x}$ & 19.972 & 7.100 & -2.936 & 0.781 & I & 10 & 0.680 & 5.611 \\
\hline${ }^{78} \mathrm{Se}$ & 9.750 & 3.920 & 12.152 & $\mathrm{x}$ & 20.931 & 9.700 & -0.938 & 0.789 & I & 9 & 2.191 & 4.500 \\
\hline${ }^{79} \mathrm{Se}$ & 9.875 & 1.655 & 8.594 & $\mathrm{x}$ & 20.757 & 7.800 & -3.382 & 0.848 & I & 5 & 0.365 & 4.500 \\
\hline${ }^{80} \mathrm{Se}$ & 10.000 & 3.680 & 9.542 & $\mathrm{~s}$ & 19.945 & 9.000 & -0.638 & 0.777 & I & 7 & 1.960 & 3.292 \\
\hline${ }^{81} \mathrm{Se}$ & 10.125 & 1.620 & 9.063 & $\mathrm{x}$ & 19.457 & 6.600 & -2.371 & 0.754 & I & 5 & 0.491 & 6.750 \\
\hline${ }^{82} \mathrm{Se}$ & 10.250 & 3.785 & 8.496 & $\mathrm{~S}$ & 19.999 & 9.000 & -0.398 & 0.771 & I & 5 & 1.735 & 4.125 \\
\hline${ }^{75} \mathrm{Br}$ & 9.375 & 1.135 & 12.500 & $\mathrm{~s}$ & 20.232 & 7.000 & -3.812 & 0.810 & $\mathrm{G}$ & 30 & 1.897 & 5.894 \\
\hline${ }^{76} \mathrm{Br}$ & 9.500 & 0.000 & 10.157 & $\mathrm{~s}$ & 17.439 & 4.474 & -3.563 & 0.735 & I & 27 & 0.688 & 8.856 \\
\hline${ }^{77} \mathrm{Br}$ & 9.625 & 1.360 & 9.634 & $\mathrm{~s}$ & 20.304 & 7.400 & -3.628 & 0.841 & I & 26 & 1.025 & 6.340 \\
\hline${ }^{78} \mathrm{Br}$ & 9.750 & 0.000 & 9.111 & $\mathrm{~s}$ & 19.027 & 5.200 & -4.186 & 0.786 & I & 25 & 0.438 & 6.833 \\
\hline${ }^{79} \mathrm{Br}$ & 9.875 & 0.900 & 8.588 & $\mathrm{~s}$ & 17.657 & 5.299 & -2.555 & 0.731 & I & 29 & 1.222 & 5.098 \\
\hline${ }^{80} \mathrm{Br}$ & 10.000 & 0.000 & 7.034 & $\mathrm{x}$ & 19.132 & 5.300 & -4.190 & 0.807 & I & 28 & 0.586 & 8.421 \\
\hline${ }^{81} \mathrm{Br}$ & 10.125 & 1.345 & 7.542 & $\mathrm{~s}$ & 18.758 & 6.200 & -2.477 & 0.764 & I & 26 & 1.372 & 5.720 \\
\hline${ }^{82} \mathrm{Br}$ & 10.250 & 0.000 & 4.620 & $\mathrm{x}$ & 16.249 & 3.900 & -2.922 & 0.731 & I & 25 & 0.988 & 6.750 \\
\hline${ }^{83} \mathrm{Br}$ & 10.375 & 1.435 & 6.496 & $\mathrm{~s}$ & 17.181 & 5.400 & -1.595 & 0.705 & I & 27 & 2.398 & 5.851 \\
\hline${ }^{76} \mathrm{Kr}$ & 9.500 & 4.185 & 14.500 & $\mathrm{~s}$ & 22.024 & 10.600 & -1.407 & 0.818 & I & 25 & 2.879 & 8.375 \\
\hline${ }^{77} \mathrm{Kr}$ & 9.625 & 0.825 & 12.157 & $\mathrm{~s}$ & 17.640 & 5.000 & -2.521 & 0.686 & I & 25 & 1.109 & 5.729 \\
\hline${ }^{78} \mathrm{Kr}$ & 9.750 & 3.725 & 11.634 & $\mathrm{~s}$ & 21.613 & 10.000 & -1.595 & 0.827 & I & 27 & 2.882 & 6.933 \\
\hline${ }^{79} \mathrm{Kr}$ & 9.875 & 1.610 & 10.375 & $\mathrm{x}$ & 21.234 & 7.700 & -3.470 & 0.823 & I & 27 & 0.984 & 6.500 \\
\hline${ }^{80} \mathrm{Kr}$ & 10.000 & 2.975 & 10.588 & $\mathrm{~s}$ & 19.325 & 7.800 & -0.926 & 0.732 & G & 25 & 3.699 & 6.195 \\
\hline${ }^{81} \mathrm{Kr}$ & 10.125 & 1.410 & 10.902 & $\mathrm{x}$ & 20.026 & 6.400 & -2.656 & 0.734 & I & 25 & 1.280 & 6.604 \\
\hline${ }^{82} \mathrm{Kr}$ & 10.250 & 3.245 & 9.542 & $\mathrm{~s}$ & 20.026 & 8.300 & -0.834 & 0.748 & I & 25 & 3.187 & 7.625 \\
\hline${ }^{83} \mathrm{Kr}$ & 10.375 & 1.730 & 9.019 & $\mathrm{~s}$ & 20.178 & 6.800 & -2.345 & 0.749 & I & 26 & 2.035 & 7.705 \\
\hline${ }^{84} \mathrm{Kr}$ & 10.500 & 3.375 & 1.919 & $\mathrm{x}$ & 19.829 & 9.500 & -1.203 & 0.908 & I & 27 & 3.587 & 8.548 \\
\hline${ }^{77} \mathrm{Rb}$ & 9.625 & 1.350 & 12.500 & $\mathrm{~S}$ & 19.237 & 6.300 & -2.722 & 0.736 & I & 7 & 0.615 & 8.333 \\
\hline${ }^{78} \mathrm{Rb}$ & 9.750 & 0.000 & 11.230 & $\mathrm{~s}$ & 12.924 & 2.200 & -1.670 & 0.536 & G & 3 & 0.256 & 3.576 \\
\hline${ }^{79} \mathrm{Rb}$ & 9.875 & 0.785 & 9.960 & $\mathrm{~s}$ & 19.589 & 6.000 & -3.451 & 0.771 & I & 3 & 0.097 & 7.250 \\
\hline${ }^{80} \mathrm{Rb}$ & 10.000 & 0.000 & 8.690 & $\mathrm{~s}$ & 8.655 & 1.000 & -0.854 & 0.448 & I & 3 & 0.236 & 0.625 \\
\hline${ }^{81} \mathrm{Rb}$ & 10.125 & 0.950 & 7.420 & $\mathrm{~s}$ & 19.439 & 6.200 & -3.216 & 0.792 & I & 7 & 0.434 & 5.271 \\
\hline${ }^{82} \mathrm{Rb}$ & 10.250 & 0.000 & 6.150 & $\mathrm{~S}$ & 20.097 & 5.700 & -4.503 & 0.832 & G & 5 & 0.192 & 3.283 \\
\hline${ }^{83} \mathrm{Rb}$ & 10.375 & 1.325 & 4.880 & $\mathrm{~s}$ & 20.403 & 7.300 & -3.344 & 0.861 & I & 7 & 0.440 & 4.167 \\
\hline${ }^{84} \mathrm{Rb}$ & 10.500 & 0.000 & 3.610 & $\mathrm{~s}$ & 15.976 & 3.700 & -2.736 & 0.721 & G & 3 & 0.464 & 5.250 \\
\hline${ }^{85} \mathrm{Rb}$ & 10.625 & 1.320 & 2.340 & $\mathrm{~s}$ & 17.935 & 6.100 & -2.214 & 0.810 & I & 19 & 1.631 & 4.146 \\
\hline
\end{tabular}

Table 5: Level density parameters calculated for new Br-Kr set. 
A.6. Modeled Cross Sections vs. Experiment 

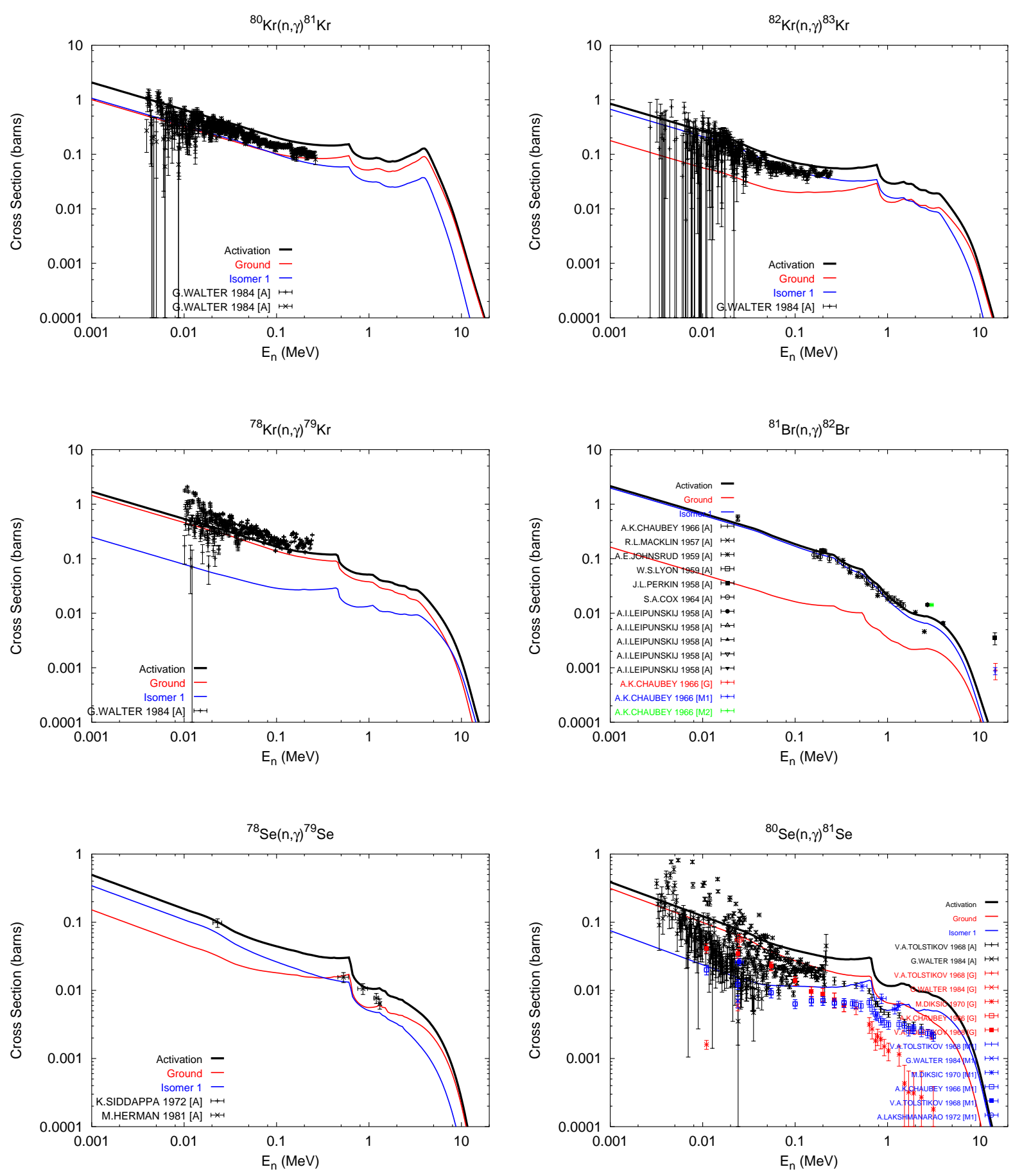

Fig. 20.- Measured vs. calculated $(\mathrm{n}, \gamma)$ cross sections. 

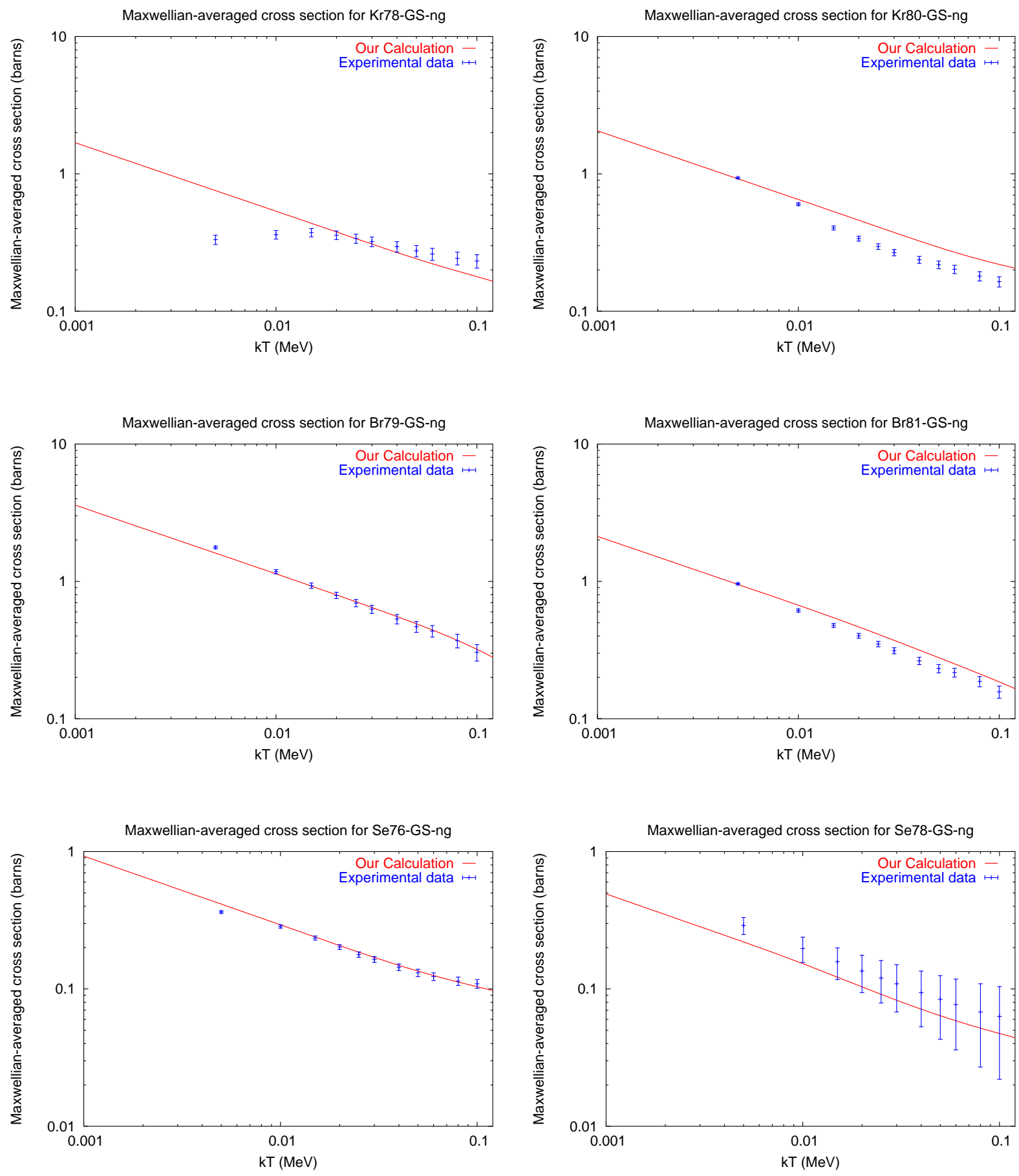

Fig. 21.- Measured vs. calculated Maxwellian averaged (n, $\gamma$ ) cross sections. 

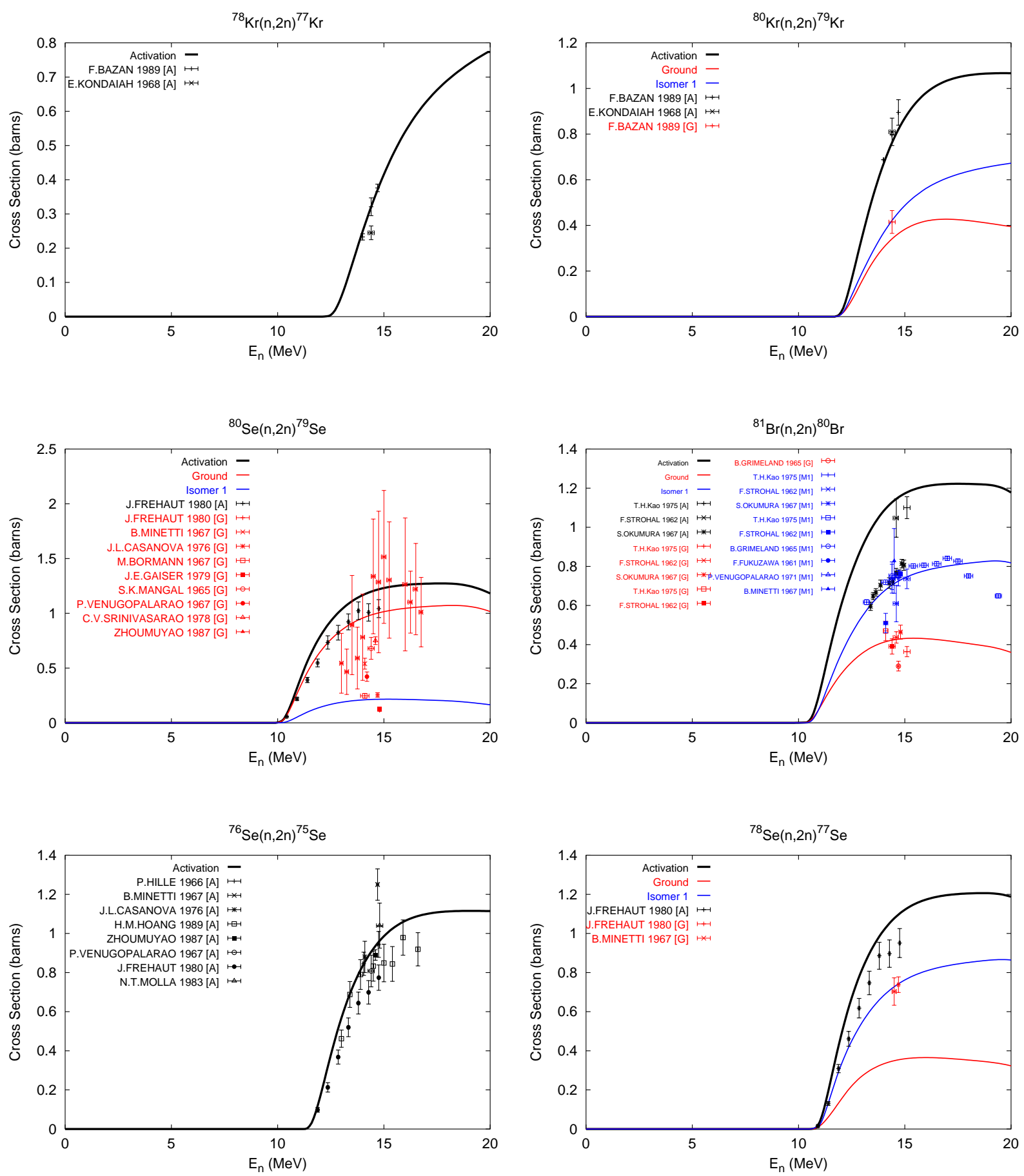

Fig. 22.- Measured vs. calculated (n,2n) cross sections. 

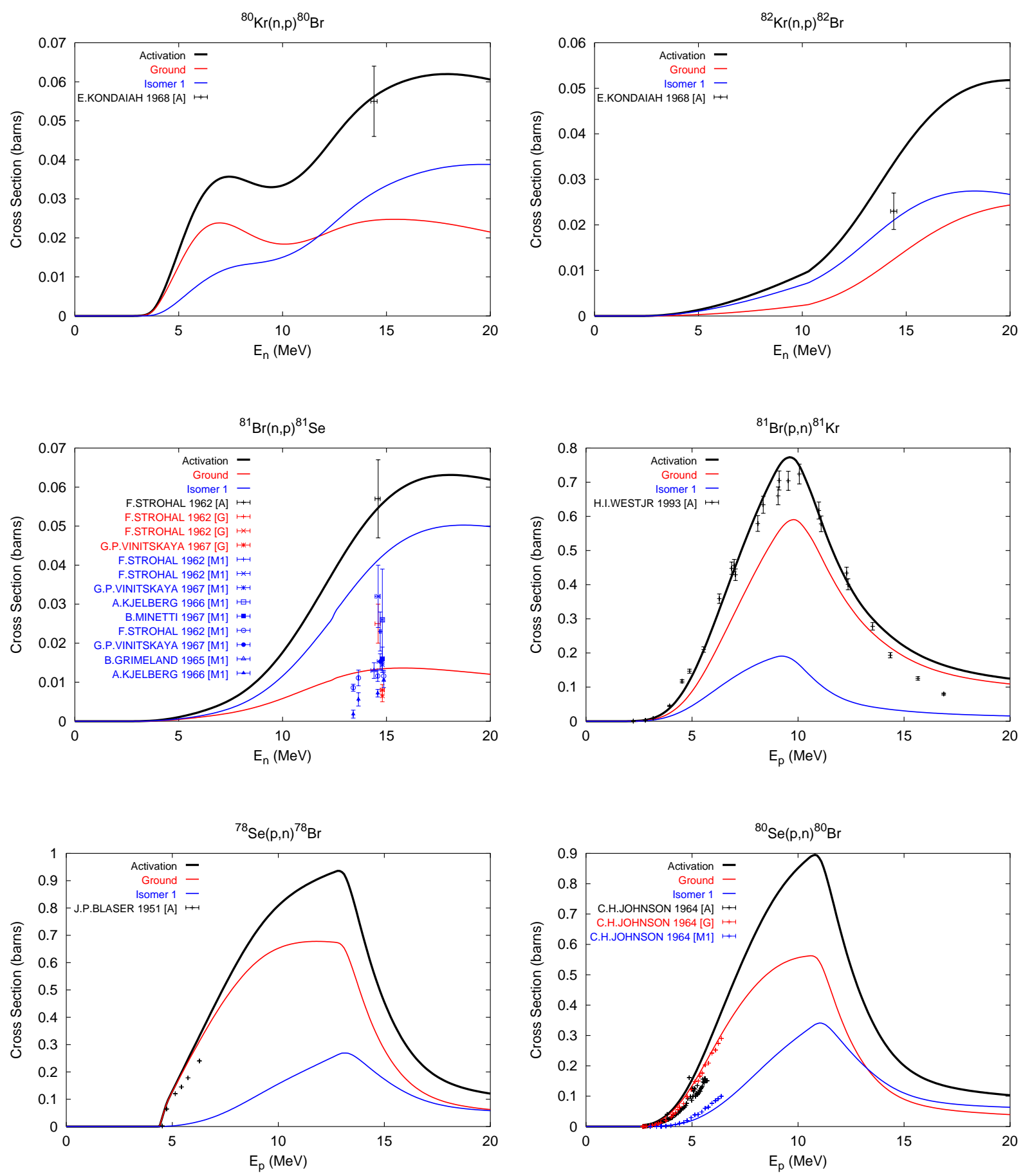

Fig. 23.- Measured vs. calculated $(\mathrm{n}, \mathrm{x})$ and $(\mathrm{p}, \mathrm{x})$ cross sections. 
A.7. Modeled Cross Sections: Production and Destruction Channels 

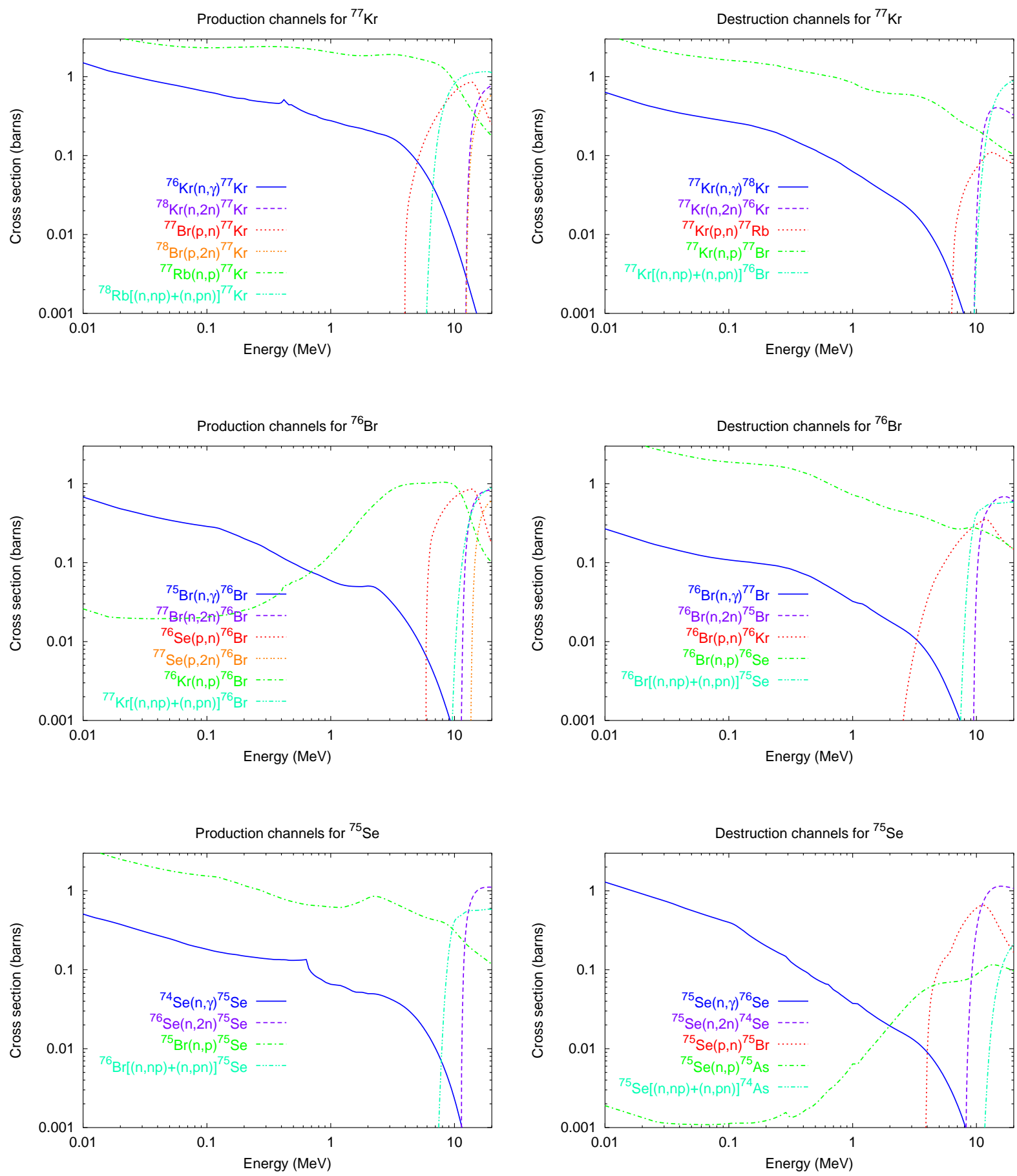

Fig. 24.- Modeled cross sections for production and destruction channels: $\mathrm{N}=41$ nuclei 

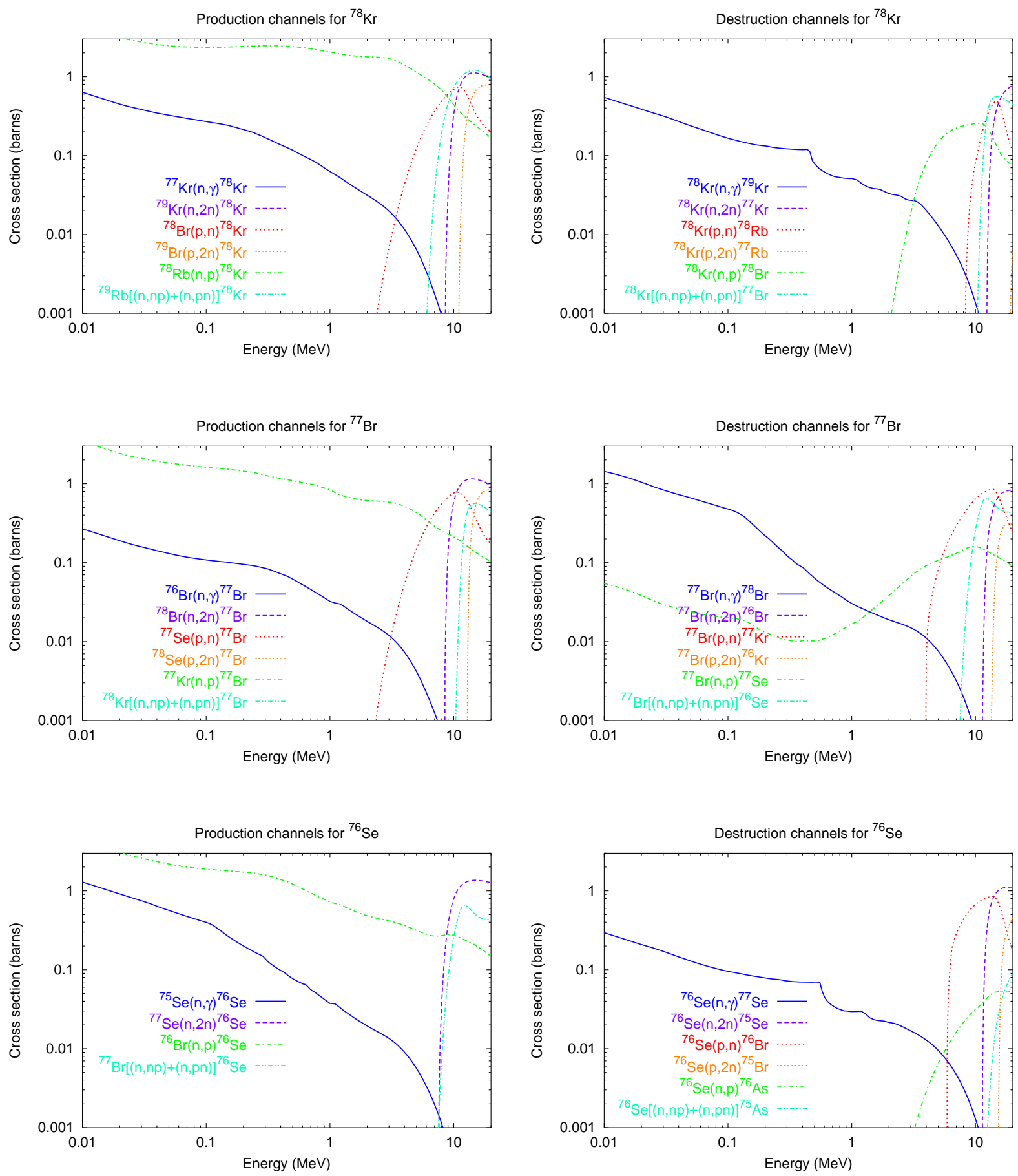

Fig. 25.- Modeled cross sections for production and destruction channels: $N=42$ nuclei 

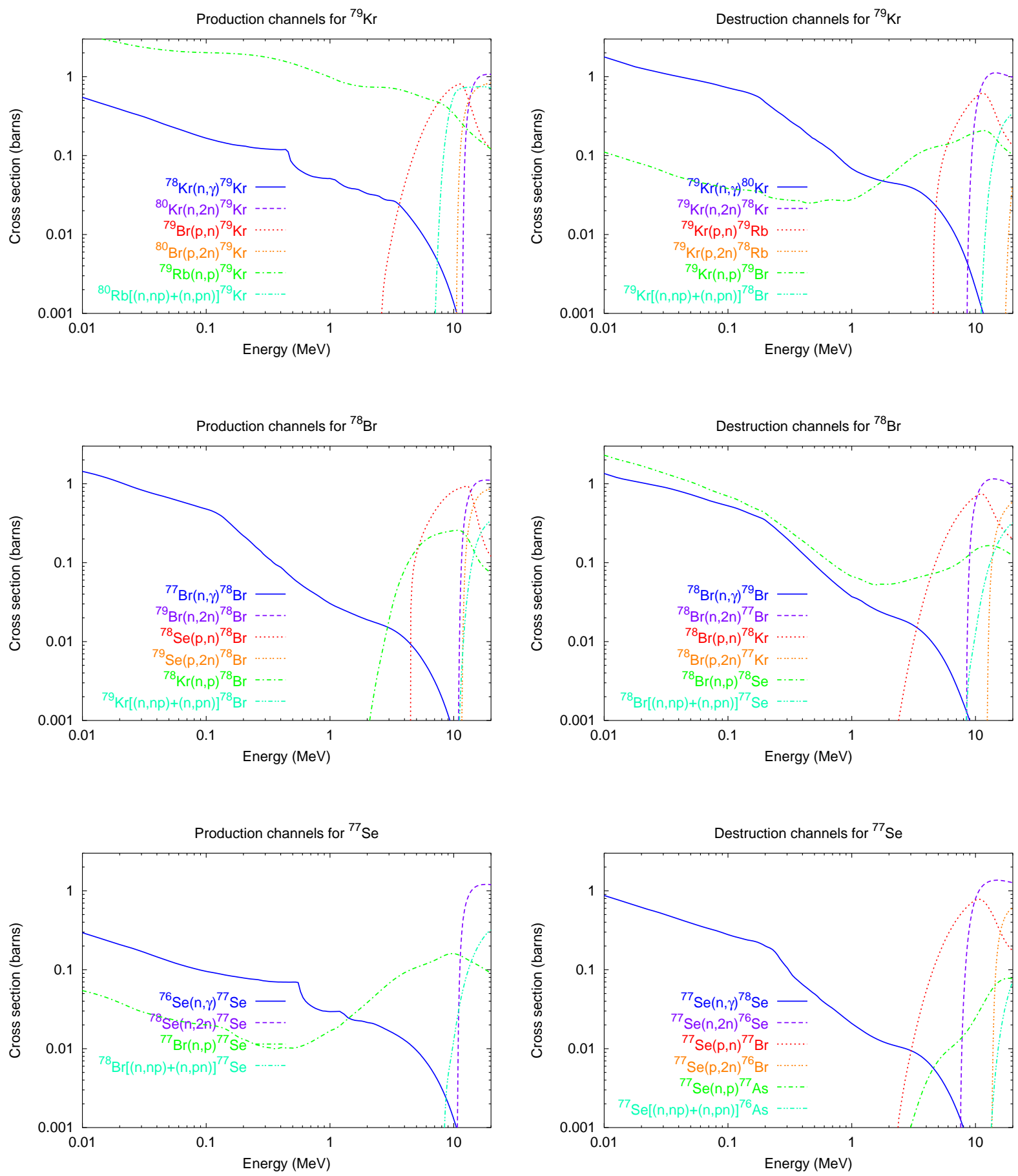

Fig. 26.- Modeled cross sections for production and destruction channels: $\mathrm{N}=43$ nuclei 

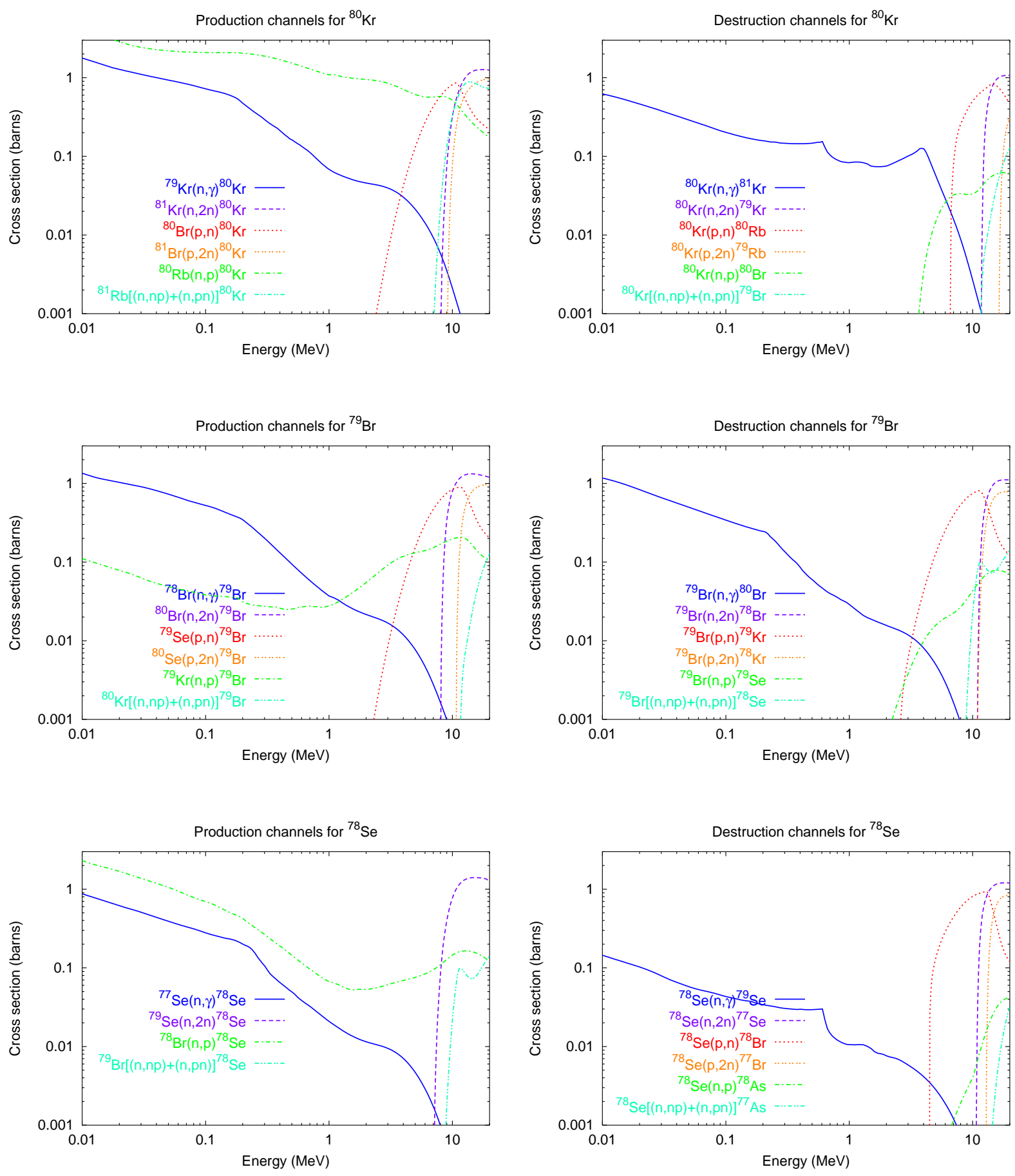

Fig. 27.- Modeled cross sections for production and destruction channels: $\mathrm{N}=44$ nuclei 

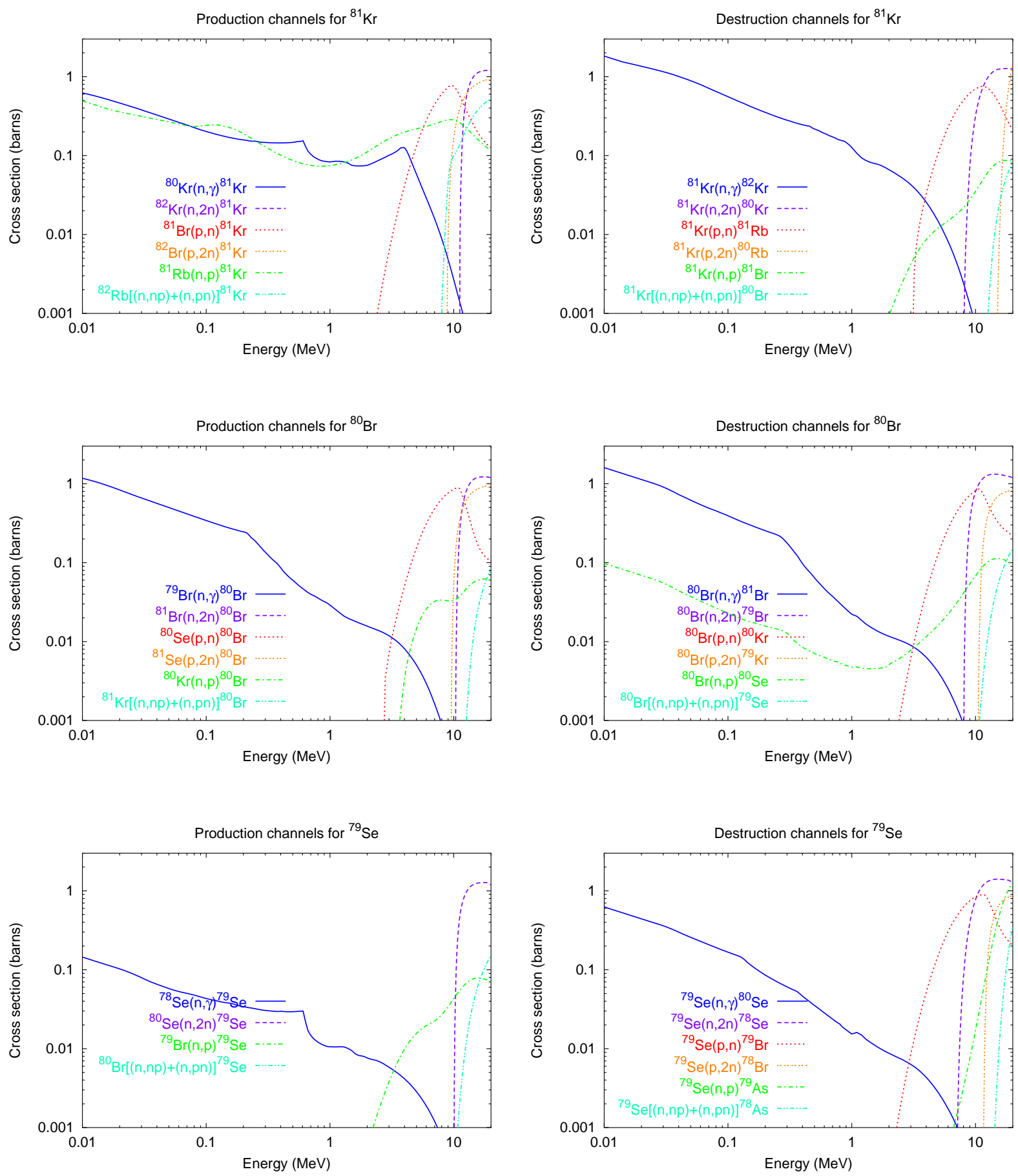

Fig. 28. - Modeled cross sections for production and destruction channels: N=45 nuclei 

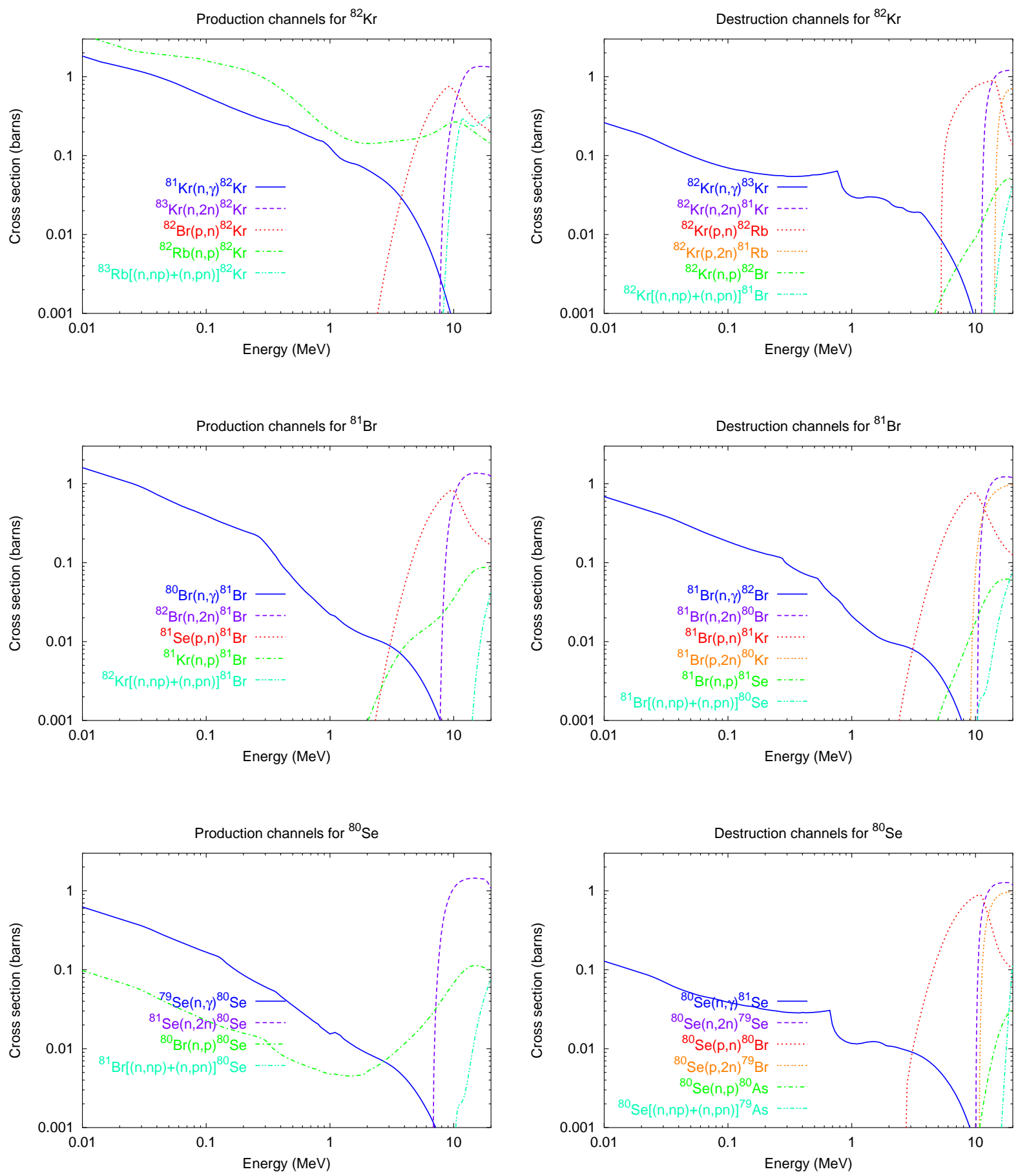

Fig. 29.- Modeled cross sections for production and destruction channels: N=46 nuclei 

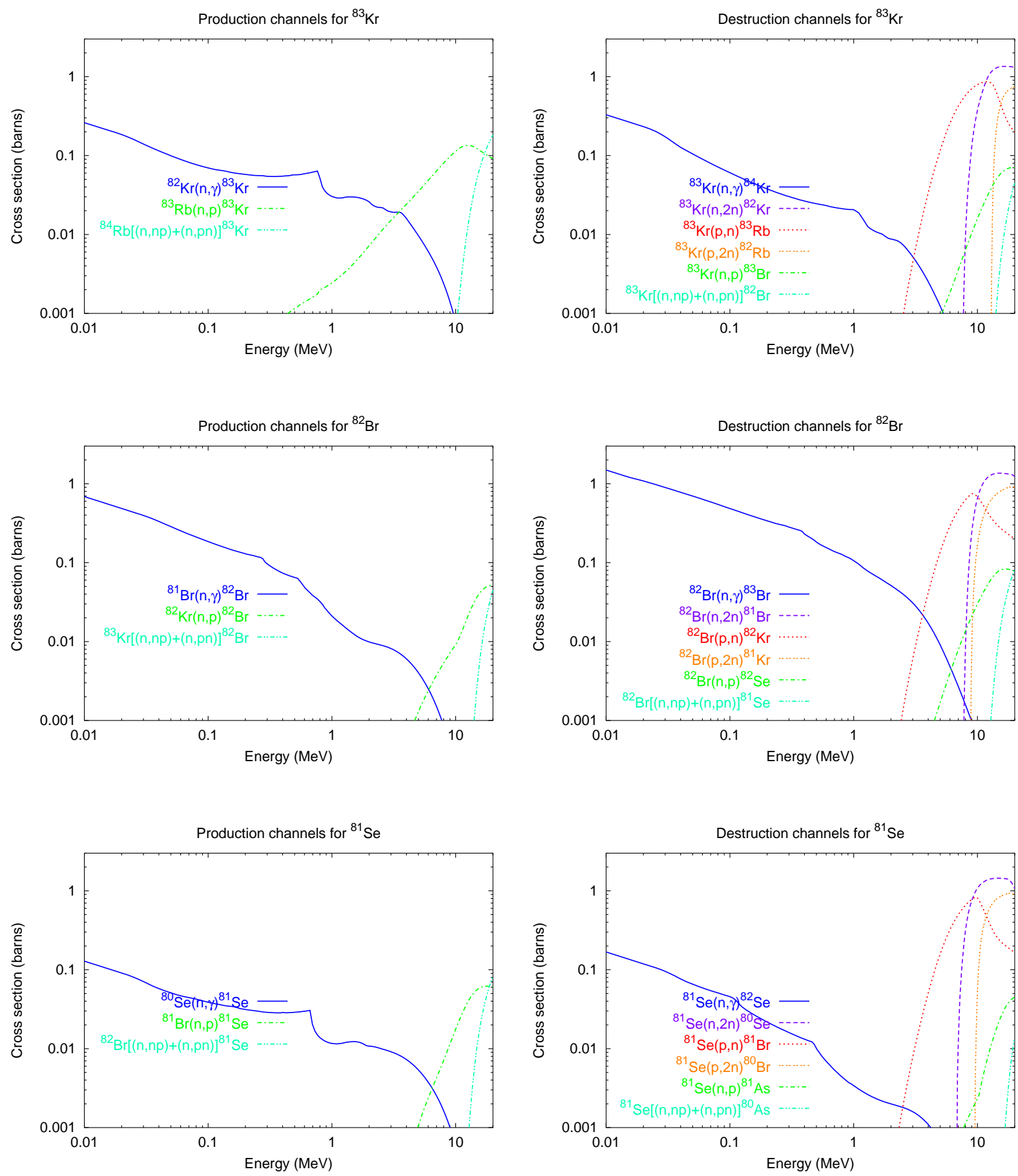

Fig. 30.- Modeled cross sections for production and destruction channels: $N=47$ nuclei 